\title{
Derivatization of Sialylated Glycopeptides (DOSG) Enabling Site-Specific Isomeric Profiling using LC-MS/MS
}

\author{
Jieqiang Zhong a, Yifan Huang a, Yehia Mechref*a \\ ${ }^{\text {a }}$ Department of Chemistry and Biochemistry, Texas Tech University, Lubbock, TX
}

79409-1061, United States.

Email: yehia.mechref@ttu.edu

Table of contents

Supplementary Experimental Section

Table S1. Results of N-glycopeptide isomeric distribution on peptide LCPDCPLLAPLNDSR (Asn156). Samples were prepared on different days as separate batches. Symbols as in Scheme 2.

Figure S1. Base peak chromatogram (ranging from m/z: 800- m/z: 2000) of non-treated tryptic fetuin fraction. The products after fractionation are mainly glycopeptides LCPDCPLLAPLNDSR $+\mathrm{Hex}_{5} \mathrm{HexNAc}_{4} \mathrm{NeuAc}_{2}$ and LCPDCPLLAPLNDSR $+\mathrm{Hex}_{6} \mathrm{HexNAc}_{5} \mathrm{NeuAc}_{3}$. Peaks appearing at 41.6min and 46.9min are glycans $\mathrm{Hex}_{5} \mathrm{HexNAc}_{4} \mathrm{NeuAc}_{2}$ and $\mathrm{Hex}_{6} \mathrm{HexNAc}_{5} \mathrm{NeuAc}_{3}$ on peptide LCPDCPLLAPLNDSR, respectively. Symbols as in Scheme 2.

Figure S2. Study of byproducts using a) full mass spectrum within the minor peak (36min-37min), and b)-e) the corresponding MS/MS spectra. The mis-labeling/under-labeling occurred on one of the residues in the peptide backbone. Symbols as in Scheme 2.

Figure S3. The MS/MS spectra of target products (a) bi-a2,3-linked sialic acids, b) mono-a2,3-linked and mono-a2,6-linked sialic acids, and c) bi-a2,6-linked sialic acids) within the major peak (37min39min). Symbols as in Scheme 2.

Figure S4. Evaluation of stability of DOSG-derivatized glycopeptides LCPDCPLLAPLNDSR+ $\mathrm{Hex}_{5} \mathrm{HexNAc}_{4} \mathrm{NeuAc}_{2}$ across time. The averaged MS spectra of retention time 37-42 $\mathrm{min}$ on a) day1, b) day 10 and c) day 20 are presented. Completely degraded products (regions labeled with red squares and magnified 10 times) were not detected. Symbols as in Scheme 2.

Figure S5. a) EIC of DOSG-derivatized O-glycopeptide TPIVGQPSIPGGPVR $+\operatorname{Hex}_{1} \operatorname{HexNAc}_{1} \mathrm{NeuAc}_{1}$, and the corresponding b) full MS spectrum and c) MS/MS spectrum. Symbols as in Scheme 2.

Figure S6. Relative quantitation of glycopeptides' isomeric distribution on a) Asn103, and b) Asn56 in tryptic digested AGP. Symbols as in Scheme 2.

Figure S7-S22. Representative MS/MS spectra of DOSG-derivatized N-glycopeptides derived from AGP. Symbols as in Scheme 2.

Figure S23-S25. The MS/MS spectra of DOSG-derivatized O-glycopeptides derived from EPO. Symbols as in Scheme 2.

\section{References}




\section{Supplementary Experimental Section}

\section{Chemicals}

Dithiothreitol (DTT), iodoacetamide (IAA), sodium bicarbonate, 1hydroxybenzotriazole monohydrate (HOBt), 1-(3-(dimethylamino) propyl)-3ethylcarbodiimide hydrochloride (EDC-HCl), methylamine (MA), fetuin from fetal calf serum (bovine fetuin), and alpha-1 acid glycoprotein from human plasma were purchased from Sigma-Aldrich (St. Louis, MO). Isopropylamine hydrochloride (iPA$\mathrm{HCl}$ ), and formic acid (FA) were obtained from Tokyo Chemical Industry (Tokyo, Japan). Recombinant human erythropoietin (EPO) was purchased from MilliporeSigma (Burlington, MA). Oasis mixed-anion exchange (MAX) cartridge was purchased from Waters (Milford, MA). C18 TopTip was purchased from Glygen (Columbia, MD). Mass spectrometry-grade trypsin/Lys-C mixture, and sequencing-grade endoprotease Glu-C were obtained from Promega (Madison, WI). HPLC-grade water was purchased from Mallinckrodt Chemicals (Phillipsburg, NJ). HPLC-grade acetonitrile (ACN) was acquired from Fisher Scientific (Fair Lawn, NJ).

\section{Tryptic Digestion of Proteins}

Tryptic digestion was performed using the protocols adapted from the work reported by Heck et al. ${ }^{1}$ and Wuhrer et al. ${ }^{2}$. In brief, fetuin, EPO, and AGP were resuspended in $50 \mathrm{mM}$ sodium bicarbonate and were put in an $80^{\circ} \mathrm{C}$ water bath for 30 min. $200 \mathrm{mM}$ of DTT was added to the denatured proteins to reach a final concentration of $5 \mathrm{mM}$, and incubation was performed in a $60^{\circ} \mathrm{C}$ water bath for $45 \mathrm{~min}$. The reduced proteins were then alkylated with $20 \mathrm{mM}$ of IAA, and incubated in a $37^{\circ} \mathrm{C}$ water bath 
for $45 \mathrm{~min}$, before quenching with using $5 \mathrm{mM}$ of DTT, and incubating at $37^{\circ} \mathrm{C}$ for 30 min. Subsequently, a trypsin/Lsy-C mixture was added in a 1:20 (enzyme: substrate) ratio to the samples, and the mixtures were incubated overnight at $37^{\circ} \mathrm{C}$.

In order to acquire the information of all of the three $\mathrm{N}$-glycosylation sites for fetuin, Glu-C digestion was added. After the tryptic digestion, fetuin samples were boiled for 10 min to quench the activity of trypsin, followed by cooling down to room temperature. Later, Glu-C was added in a 1:50 (enzyme: substrate) ratio to the fetuin samples, and the mixtures were incubated at $37^{\circ} \mathrm{C}$ overnight. The resulting peptides and glycopeptides were subjected to glycopeptide enrichment either using MAX cartridge (see supporting information for details) or fractionation.

\section{Enrichment of Glycopeptides using MAX}

The enrichment method was adapted from the work of Zhang et $\mathrm{al}^{3}$. In short, the MAX cartridges were individually conditioned three times with $1 \mathrm{~mL}$ of $\mathrm{ACN}, 100 \mathrm{mM}$ triethylammonium acetate, water and finally $95 \% \mathrm{ACN}(\mathrm{v} / \mathrm{v}), 1 \% \mathrm{TFA}(\mathrm{v} / \mathrm{v})$. The tryptic digested samples were then resuspended in $1 \mathrm{~mL}$ of $95 \% \mathrm{ACN}(\mathrm{v} / \mathrm{v}), 1 \% \mathrm{TFA}(\mathrm{v} / \mathrm{v})$ before the mixtures were loaded and washed three times with $95 \%$ ACN (v/v), 1\%TFA (v/v). Lastly, the glycopeptides were eluted with $400 \mu \mathrm{L}$ of $50 \%$ ACN (v/v), $0.1 \% \mathrm{TFA}(\mathrm{v} / \mathrm{v})$

\section{C18 Clean-up}

The protocol was adapted from the vendor's instruction. Firstly, C18 toptips were equilibrated three times with $50 \mu \mathrm{L}$ of $60 \% \mathrm{ACN}(\mathrm{v} / \mathrm{v}), 0.1 \% \mathrm{FA}(\mathrm{v} / \mathrm{v})$, and water, $0.1 \%$ FA (v/v). Subsequently, the neutralized samples were loaded on toptips twice, followed 
by washing three times with $50 \mu \mathrm{L}$ of water, $0.1 \% \mathrm{FA}(\mathrm{v} / \mathrm{v})$. Finally, bound derivatized glycopeptides were eluted three times with $50 \mu \mathrm{L}$ of $60 \% \mathrm{ACN}(\mathrm{v} / \mathrm{v}), 0.1 \% \mathrm{FA}(\mathrm{v} / \mathrm{v})$. All of the solvents were spun down using a centrifuge set at $1 \times 10^{3} \mathrm{~g}$ for $2 \mathrm{~min}$. 
Table S1. Results of N-glycopeptide isomeric distribution on peptide LCPDCPLLAPLNDSR (Asn156). Samples were prepared on different days as separate batches. Symbols as in Scheme 2.

\begin{tabular}{|c|c|c|c|c|c|c|c|c|c|}
\hline & & $\begin{array}{l}\% \text { relative } \\
\text { abundance } \\
\text { in batch } 1\end{array}$ & $\begin{array}{l}\% \text { relative } \\
\text { abundance } \\
\text { in batch } 2\end{array}$ & $\begin{array}{c}\% \text { relative } \\
\text { abundance } \\
\text { in batch } 3\end{array}$ & $\begin{array}{c}\text { \% relative } \\
\text { abundance } \\
\text { in batch } 4\end{array}$ & $\begin{array}{l}\% \text { relative } \\
\text { abundance } \\
\text { in batch } 5\end{array}$ & $\begin{array}{l}\% \text { relative } \\
\text { abundance } \\
\text { in batch } 6\end{array}$ & $\begin{array}{c}\text { \% relative } \\
\text { abundance } \\
\text { in batch } 7\end{array}$ & Avg $\pm s d$ \\
\hline \multirow{2}{*}{ 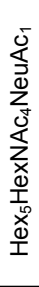 } & $\begin{array}{c}\vdots \\
\vdots \vdots \\
\vdots \\
\text { PEP }\end{array}$ & $0.40 \%$ & $0.30 \%$ & $0.10 \%$ & $0.20 \%$ & $0.20 \%$ & $0.10 \%$ & $0.20 \%$ & $0.2 \% \pm 0.1 \%$ \\
\hline & $\begin{array}{c}\# \\
\vdots \\
\vdots \\
\vdots \\
\text { PEP }\end{array}$ & $0.60 \%$ & $0.50 \%$ & $0.50 \%$ & $0.70 \%$ & $0.50 \%$ & $0.60 \%$ & $0.50 \%$ & $0.5 \% \pm 0.1 \%$ \\
\hline \multirow{3}{*}{ 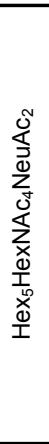 } & $\begin{array}{c}++ \\
\vdots \\
\vdots \\
\vdots \\
\text { PEP }\end{array}$ & $2.50 \%$ & $1.70 \%$ & $1.60 \%$ & $1.40 \%$ & $1.80 \%$ & $1.70 \%$ & $1.70 \%$ & $1.8 \% \pm 0.3 \%$ \\
\hline & $\begin{array}{c}+{ }^{+} \\
\vdots \\
\vdots \\
\text { PÉP }\end{array}$ & $18.40 \%$ & $17.00 \%$ & $16.30 \%$ & $16.00 \%$ & $16.10 \%$ & $16.10 \%$ & $16.30 \%$ & $16.6 \% \pm 0.9 \%$ \\
\hline & $\begin{array}{l}\# \# \\
\because: \# \\
\vdots \\
\vdots \\
\text { PEP }\end{array}$ & $9.80 \%$ & $10.30 \%$ & $9.50 \%$ & $13.30 \%$ & $9.40 \%$ & $9.60 \%$ & $9.80 \%$ & $10 \% \pm 1 \%$ \\
\hline \multirow{3}{*}{ 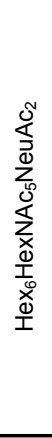 } & 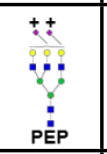 & $0.70 \%$ & $0.80 \%$ & $0.60 \%$ & $1.20 \%$ & $0.50 \%$ & $0.40 \%$ & $0.40 \%$ & $0.7 \% \pm 0.3 \%$ \\
\hline & 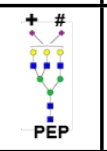 & $3.40 \%$ & $2.90 \%$ & $2.60 \%$ & $2.70 \%$ & $2.90 \%$ & $2.10 \%$ & $2.40 \%$ & $2.7 \% \pm 0.4 \%$ \\
\hline & 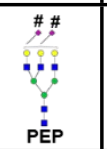 & $1.90 \%$ & $1.70 \%$ & $1.60 \%$ & $1.60 \%$ & $1.30 \%$ & $1.30 \%$ & $1.30 \%$ & $1.5 \% \pm 0.2 \%$ \\
\hline \multirow{4}{*}{ 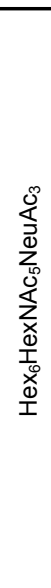 } & $\begin{array}{c}++ \pm \\
\vdots \\
\vdots \\
\vdots \\
\text { PEPP }\end{array}$ & $2.90 \%$ & $1.80 \%$ & $1.80 \%$ & $2.10 \%$ & $1.70 \%$ & $1.90 \%$ & $1.80 \%$ & $2 \% \pm 0.4 \%$ \\
\hline & $\begin{array}{c}\#++ \\
\vdots \\
\vdots \\
\text { PEP }\end{array}$ & $24.80 \%$ & $27.30 \%$ & $28.70 \%$ & $25.20 \%$ & $28.40 \%$ & $28.30 \%$ & $27.40 \%$ & $27 \% \pm 1 \%$ \\
\hline & \begin{tabular}{c}
$* \pm+$ \\
\hdashline \\
$\vdots$ \\
$\vdots$ \\
PEP
\end{tabular} & $19.70 \%$ & $20.70 \%$ & $21.70 \%$ & $19.90 \%$ & $21.60 \%$ & $22.20 \%$ & $22.20 \%$ & $21 \% \pm 1 \%$ \\
\hline & 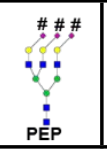 & $3.10 \%$ & $3.10 \%$ & $3.20 \%$ & $3.40 \%$ & $2.70 \%$ & $3.30 \%$ & $3.40 \%$ & $3.2 \% \pm 0.2 \%$ \\
\hline \multirow{2}{*}{ 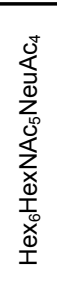 } & 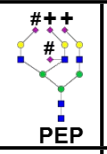 & $7.20 \%$ & $7.10 \%$ & $7.10 \%$ & $7.30 \%$ & $7.60 \%$ & $7.70 \%$ & $7.60 \%$ & $7.4 \% \pm 0.3 \%$ \\
\hline & 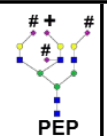 & $4.60 \%$ & $4.80 \%$ & $4.60 \%$ & $5.00 \%$ & $5.10 \%$ & $4.60 \%$ & $5.00 \%$ & $4.8 \% \pm 0.2 \%$ \\
\hline
\end{tabular}




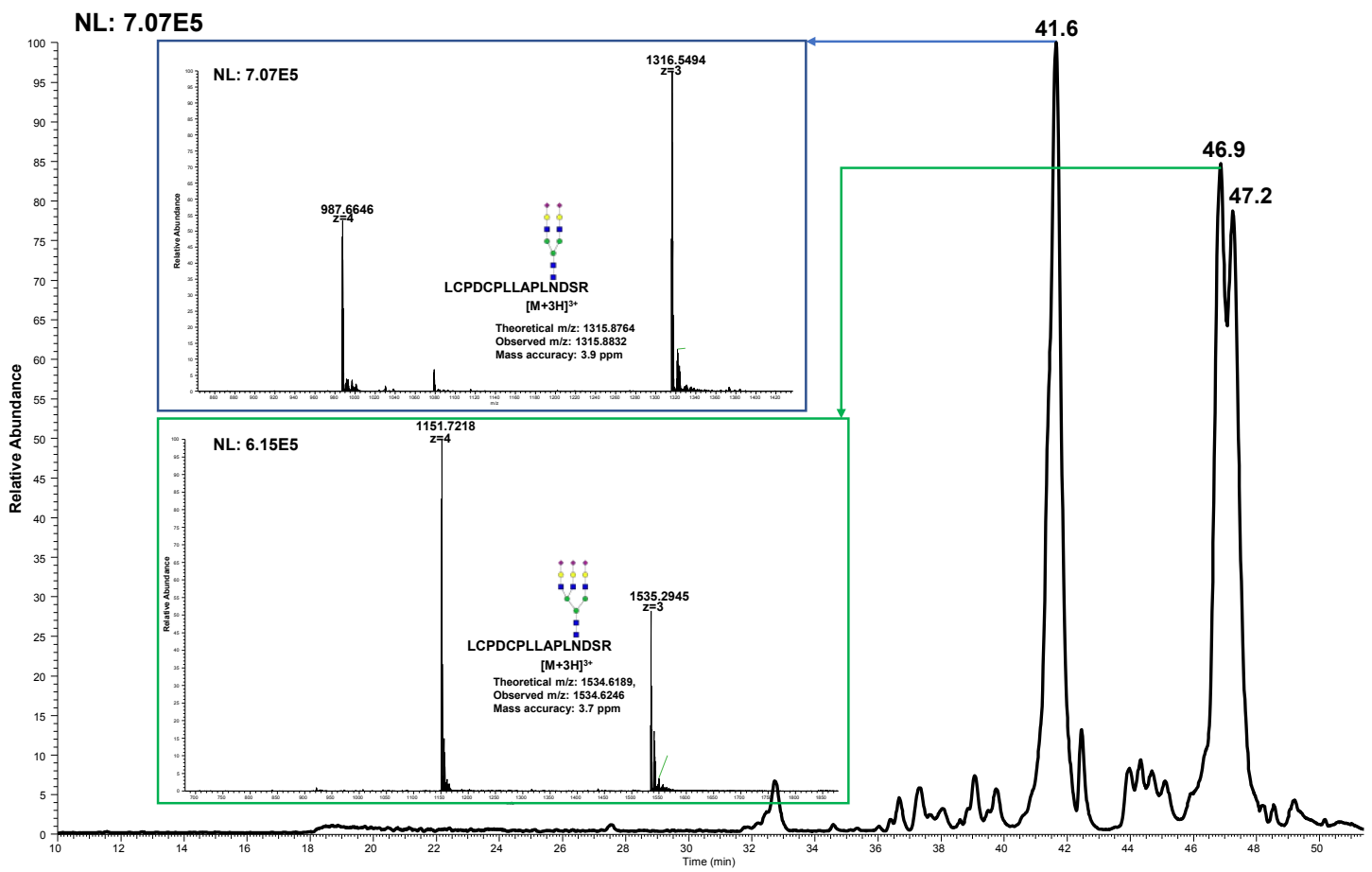

Figure S1. Base peak chromatogram (ranging from m/z: 800- m/z: 2000) of non-treated tryptic fetuin fraction. The products after fractionation are mainly glycopeptides LCPDCPLLAPLNDSR $+\mathrm{Hex}_{5} \mathrm{HexNAc}_{4} \mathrm{NeuAc}_{2}$ and LCPDCPLLAPLNDSR + $\mathrm{Hex}_{6} \mathrm{HexNAc}_{5} \mathrm{NeuAc}_{3}$. Peaks appearing at $41.6 \mathrm{~min}$ and $46.9 \mathrm{~min}$ are glycans $\mathrm{Hex}_{5} \mathrm{HexNAc}_{4} \mathrm{NeuAc}_{2}$ and $\mathrm{Hex}_{6} \mathrm{HexNAc}_{5} \mathrm{NeuAc}_{3}$ on peptide LCPDCPLLAPLNDSR, respectively. Symbols as in Scheme 2. 


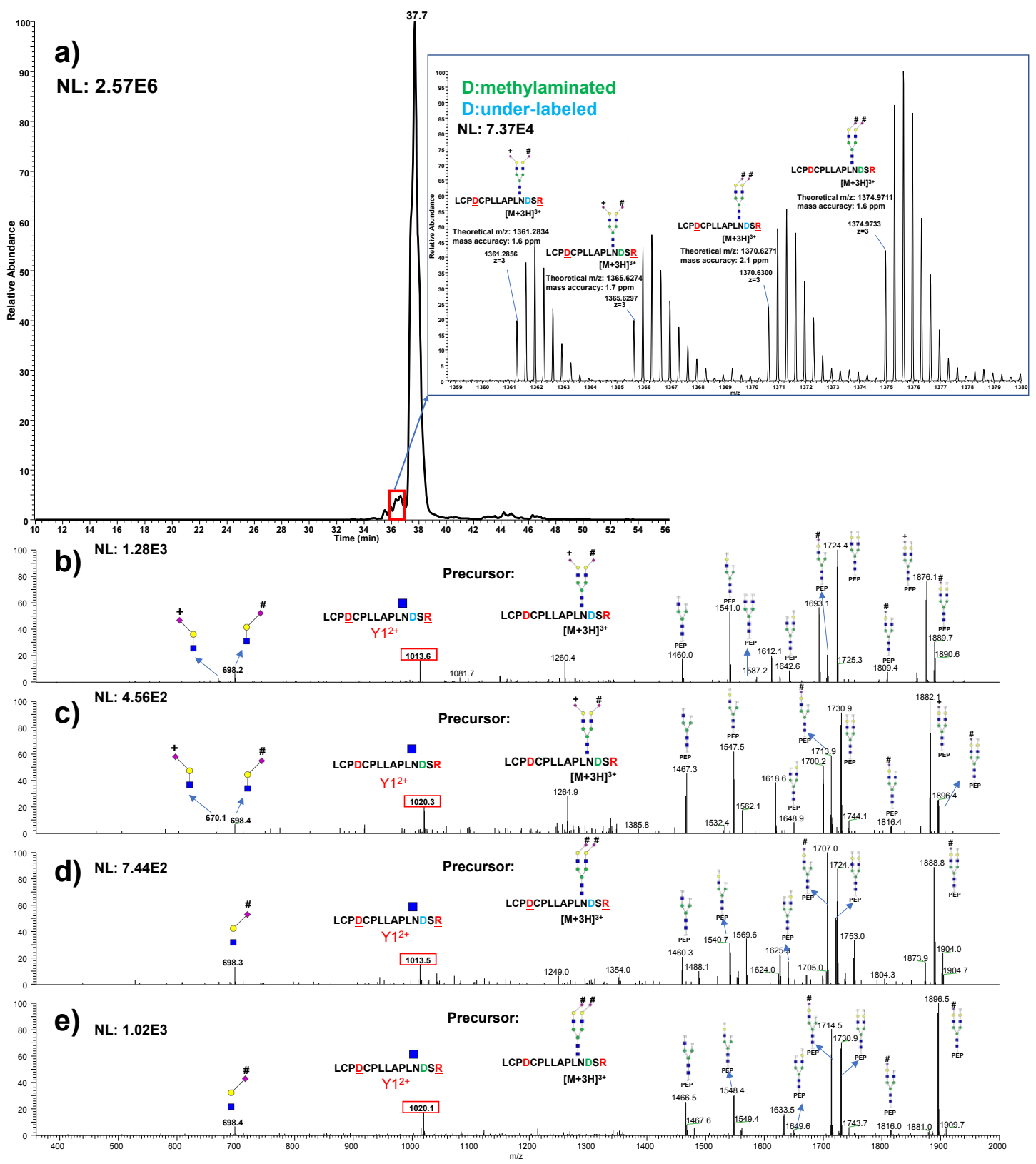

Figure S2. Study of byproducts using a) EIC of the target glycopeptides. Inset is the full mass spectrum within the minor peak (36min-37min), and b)-e) the corresponding MS/MS spectra. The mis-labeling/under-labeling occurred on one of the residues in the peptide backbone. Symbols as in Scheme 2. 


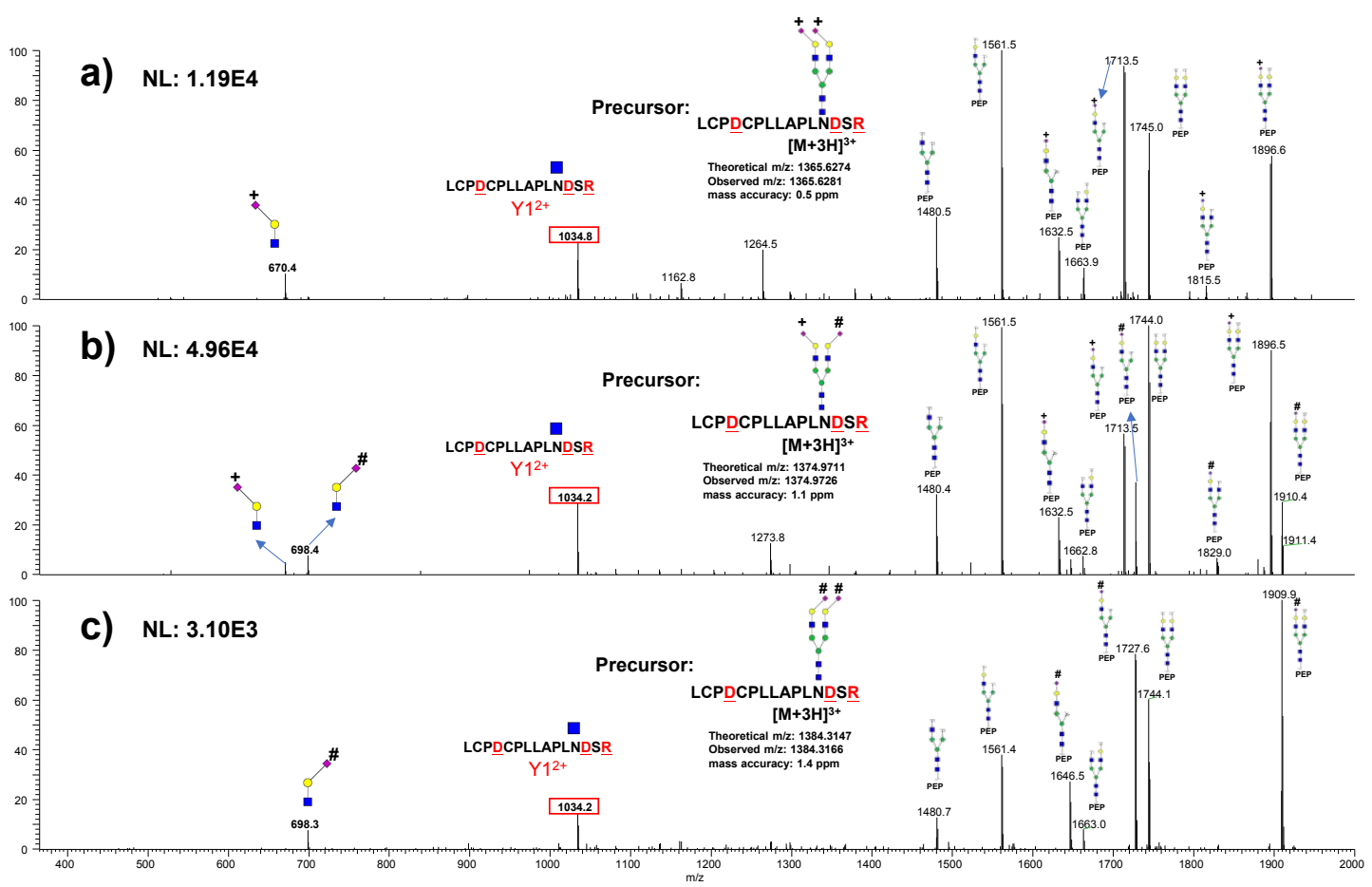

Figure S3. The MS/MS spectra of target products (a) bi-a2,3-linked sialic acids, b) mono-a2,3-linked and mono-a2,6-linked sialic acids, and c) bi-a2,6-linked sialic acids) within the major peak (37min-39min). Symbols as in Scheme 2. 


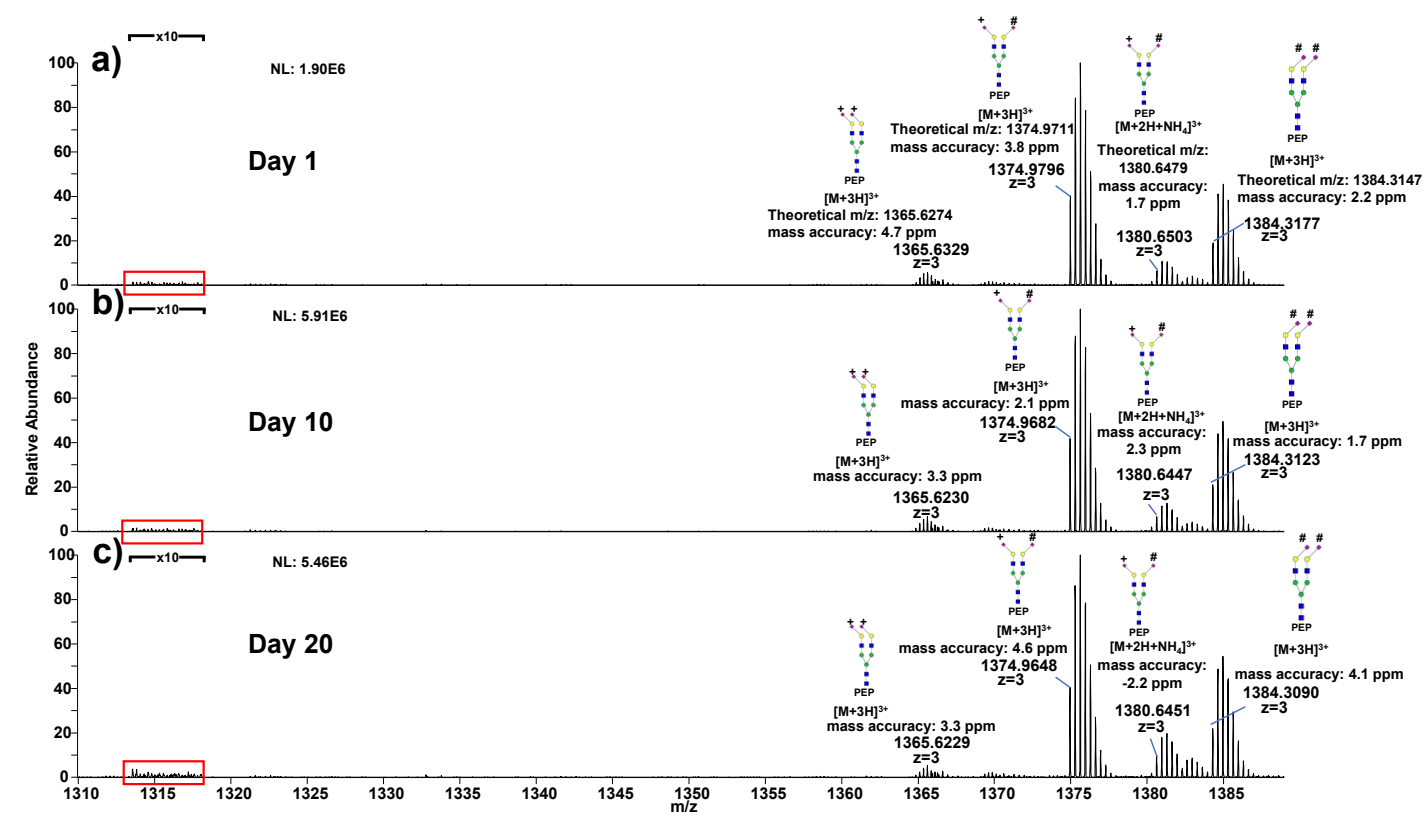

Figure S4. Evaluation of stability of DOSG-derivatized glycopeptides LCPDCPLLAPLNDSR $+\mathrm{Hex}_{5} \mathrm{HexNAc}_{4} \mathrm{NeuAc}_{2}$ across time. The averaged MS spectra of retention time 37-42 min on a) day 1 , b) day 10 and c) day 20 are presented. Completely degraded products (regions labeled with red squares and magnified 10 times) were not detected. Symbols as in Scheme 2. 


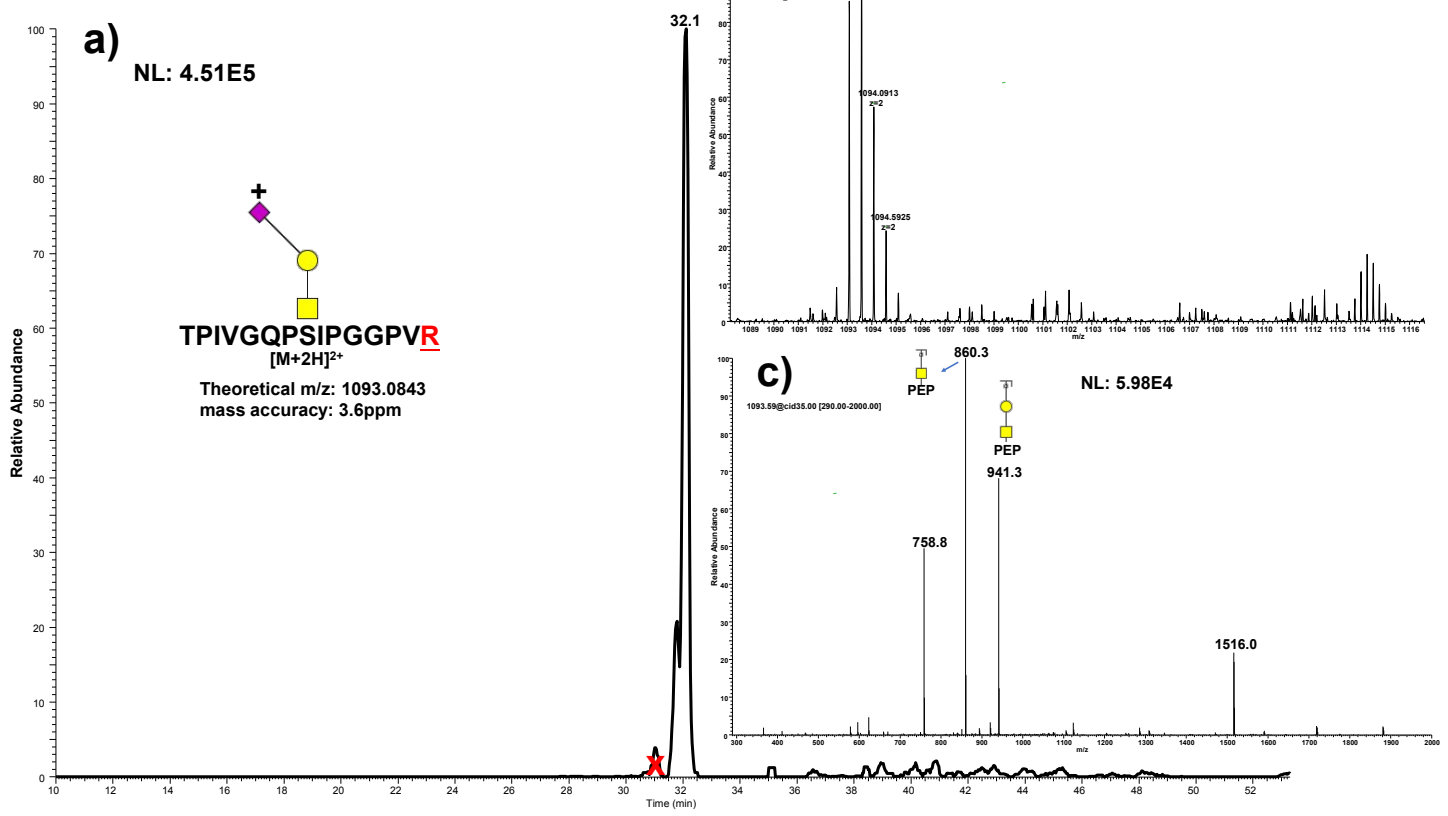

Figure S5. a) EIC of DOSG-derivatized O-glycopeptide TPIVGQPSIPGGPVR+ $\mathrm{Hex}_{1} \mathrm{HexNAc}_{1} \mathrm{NeuAc}_{1}$, and the corresponding b) full MS spectrum and c) MS/MS spectrum. Symbols as in Scheme 2. 

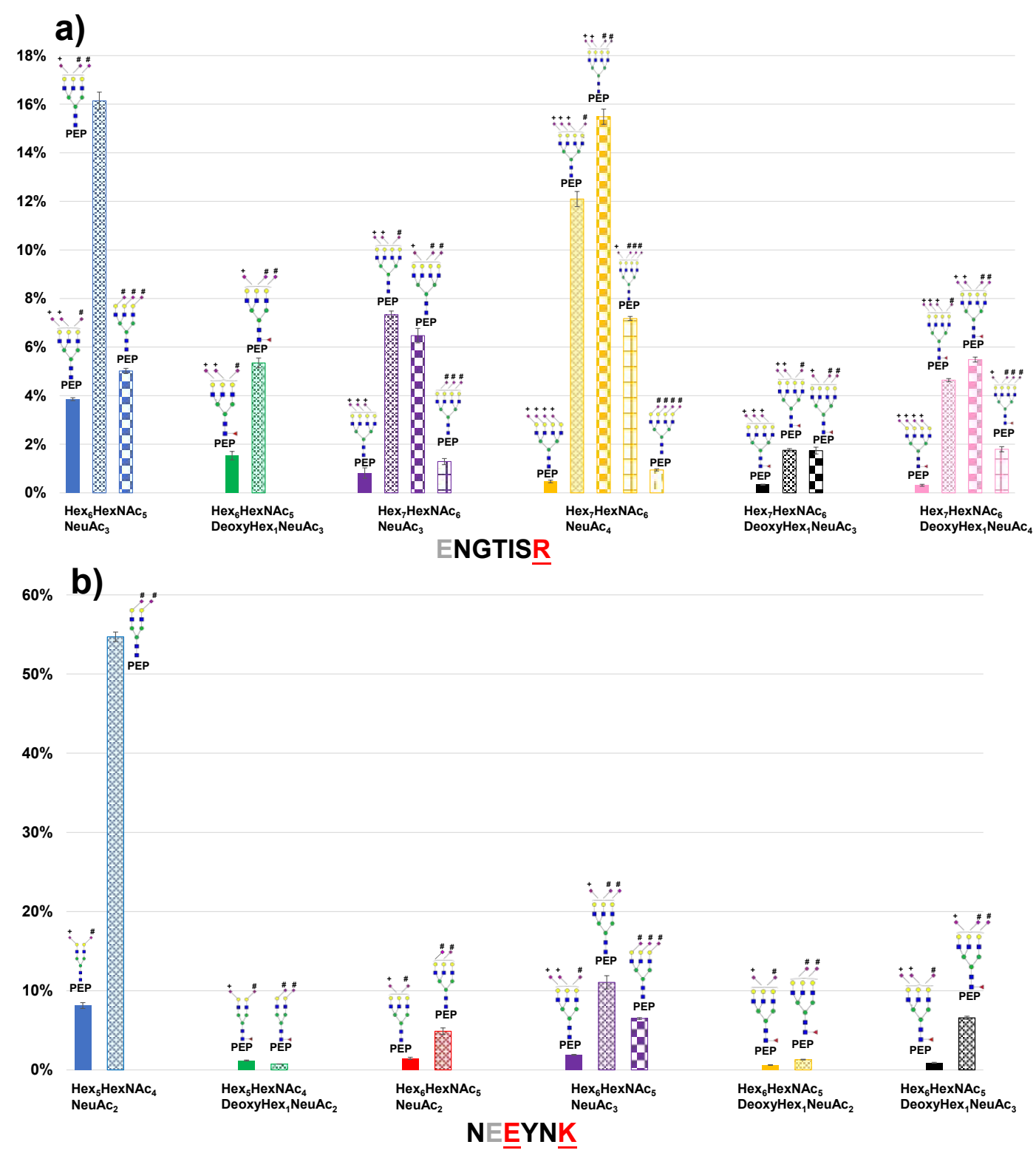

Figure S6. Relative quantitation of glycopeptides' isomeric distribution on a) Asn103, and b) Asn56 in tryptic digested AGP. Symbols as in Scheme 2. 


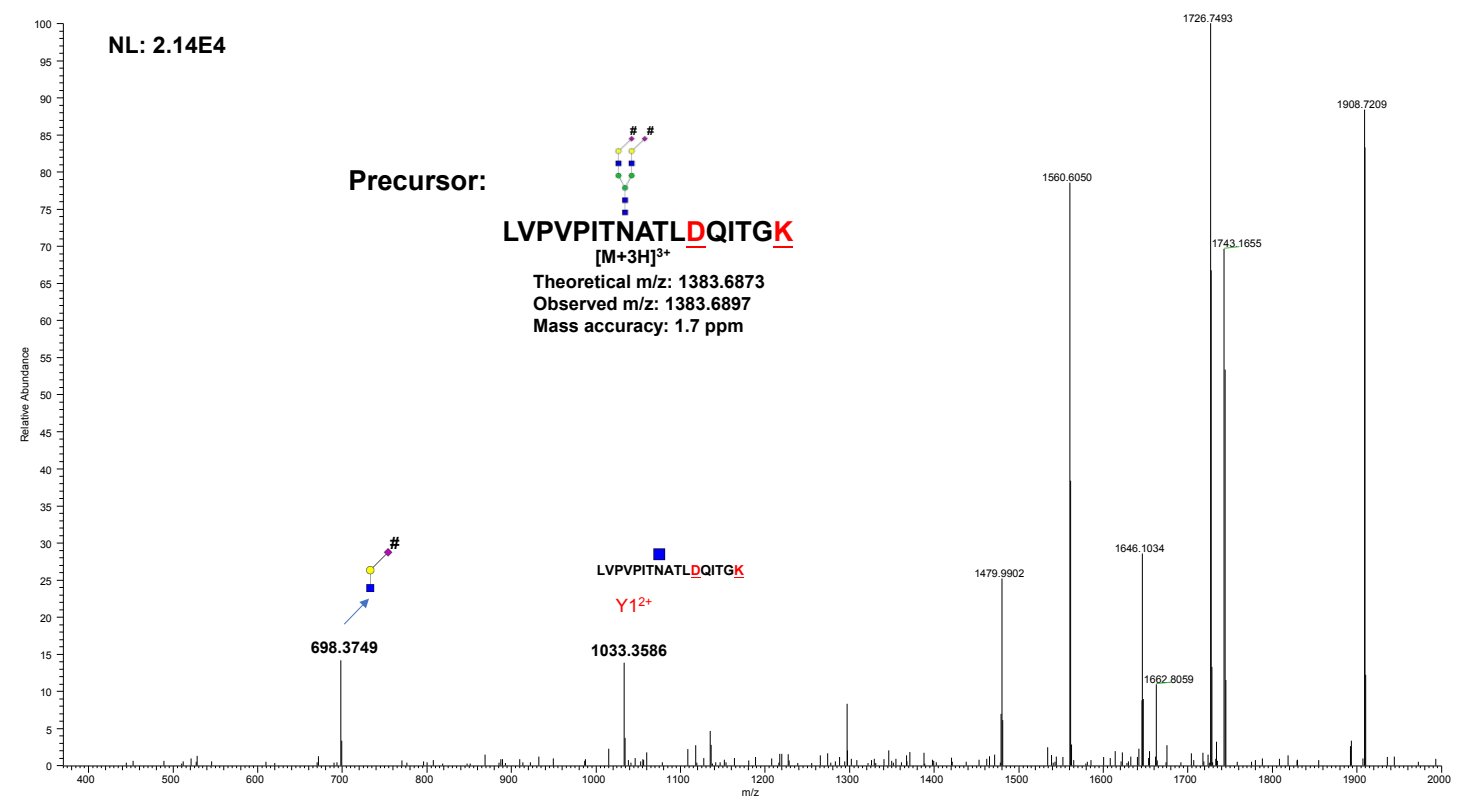

Figure S7. Representative MS/MS spectrum DOSG-derivatized of N-glycopeptides derived from AGP. Symbols as in Scheme 2. 


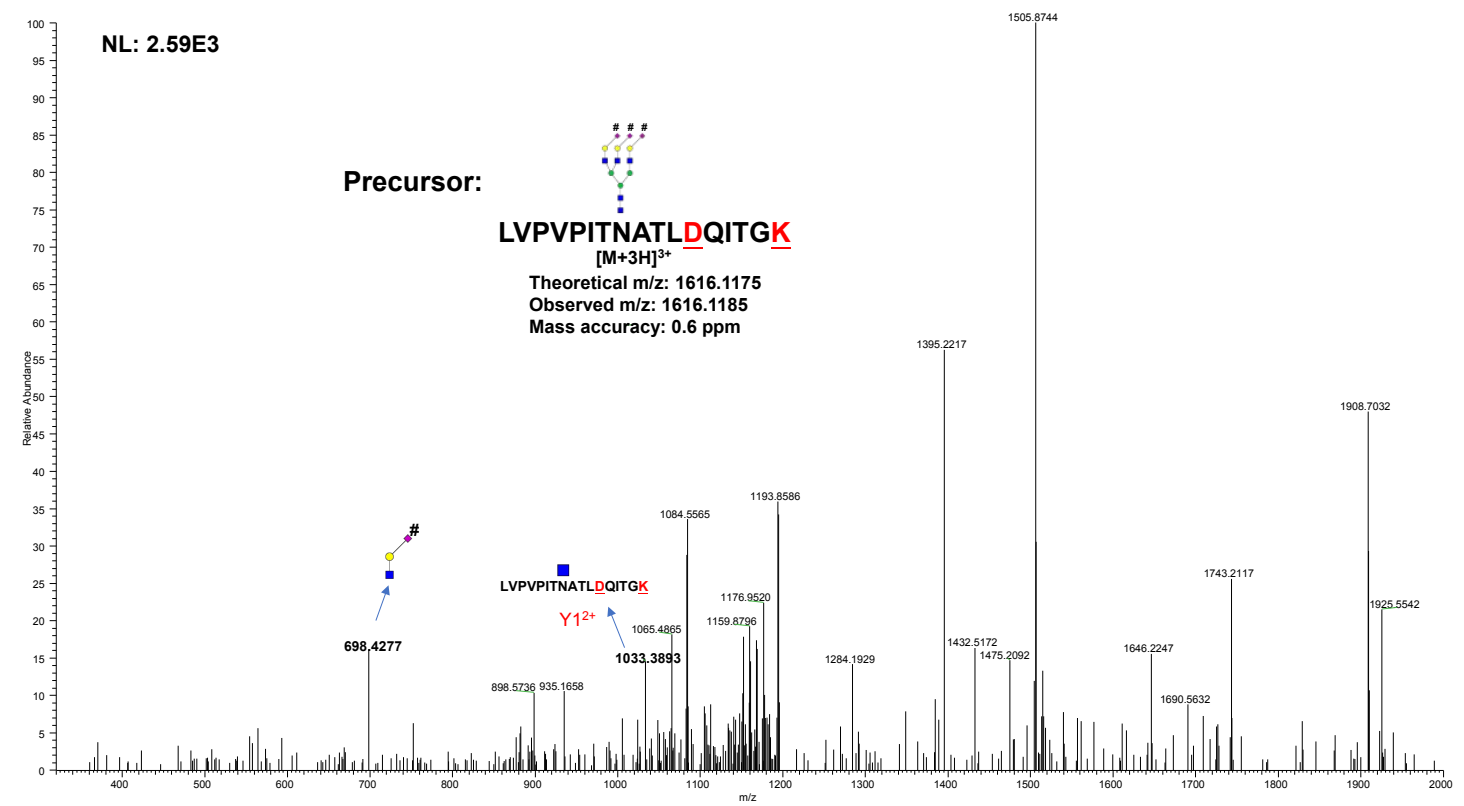

Figure S8. Representative MS/MS spectrum of DOSG-derivatized N-glycopeptides derived from AGP. Symbols as in Scheme 2. 


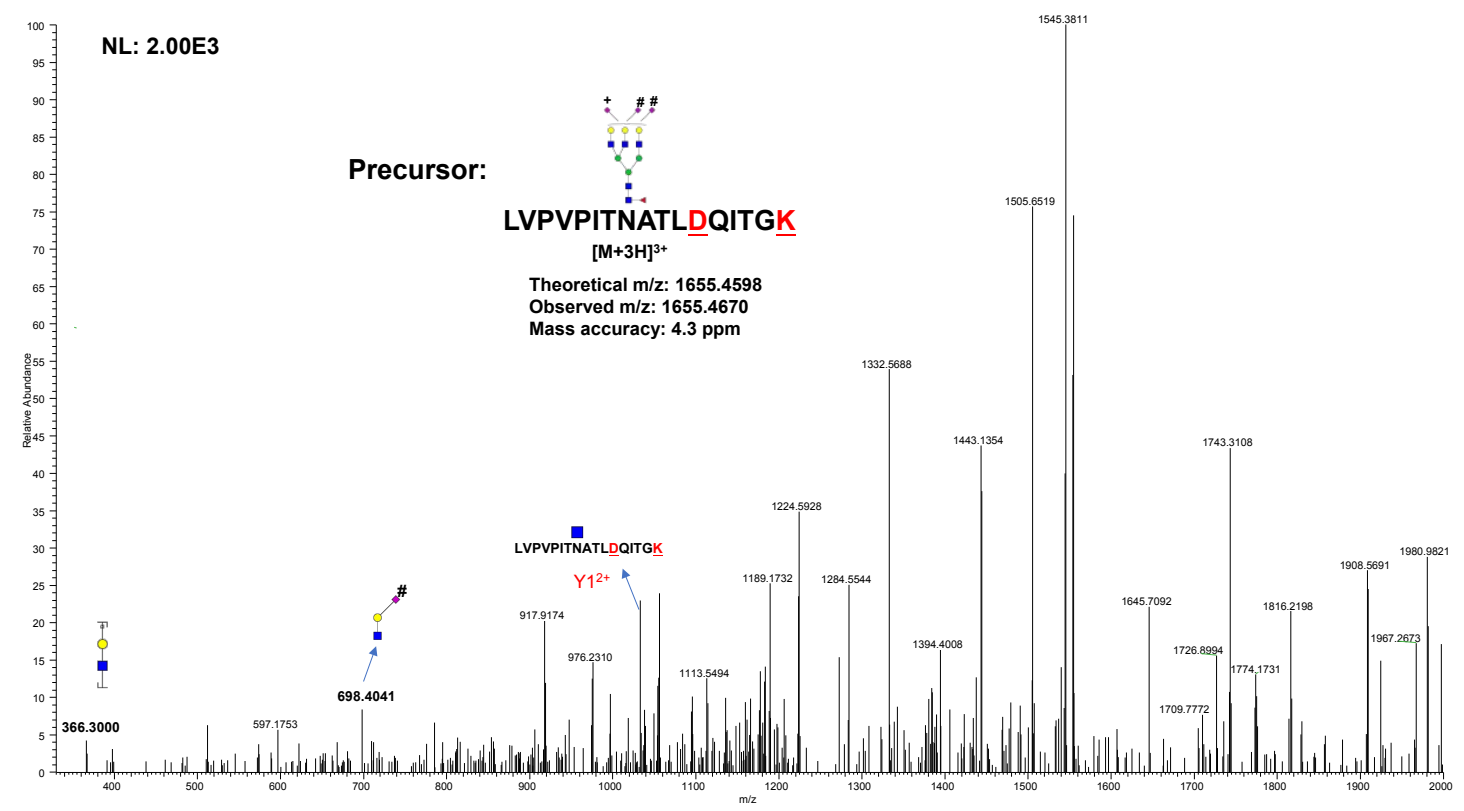

Figure S9. Representative MS/MS spectrum of DOSG-derivatized N-glycopeptides derived from AGP. Symbols as in Scheme 2. 


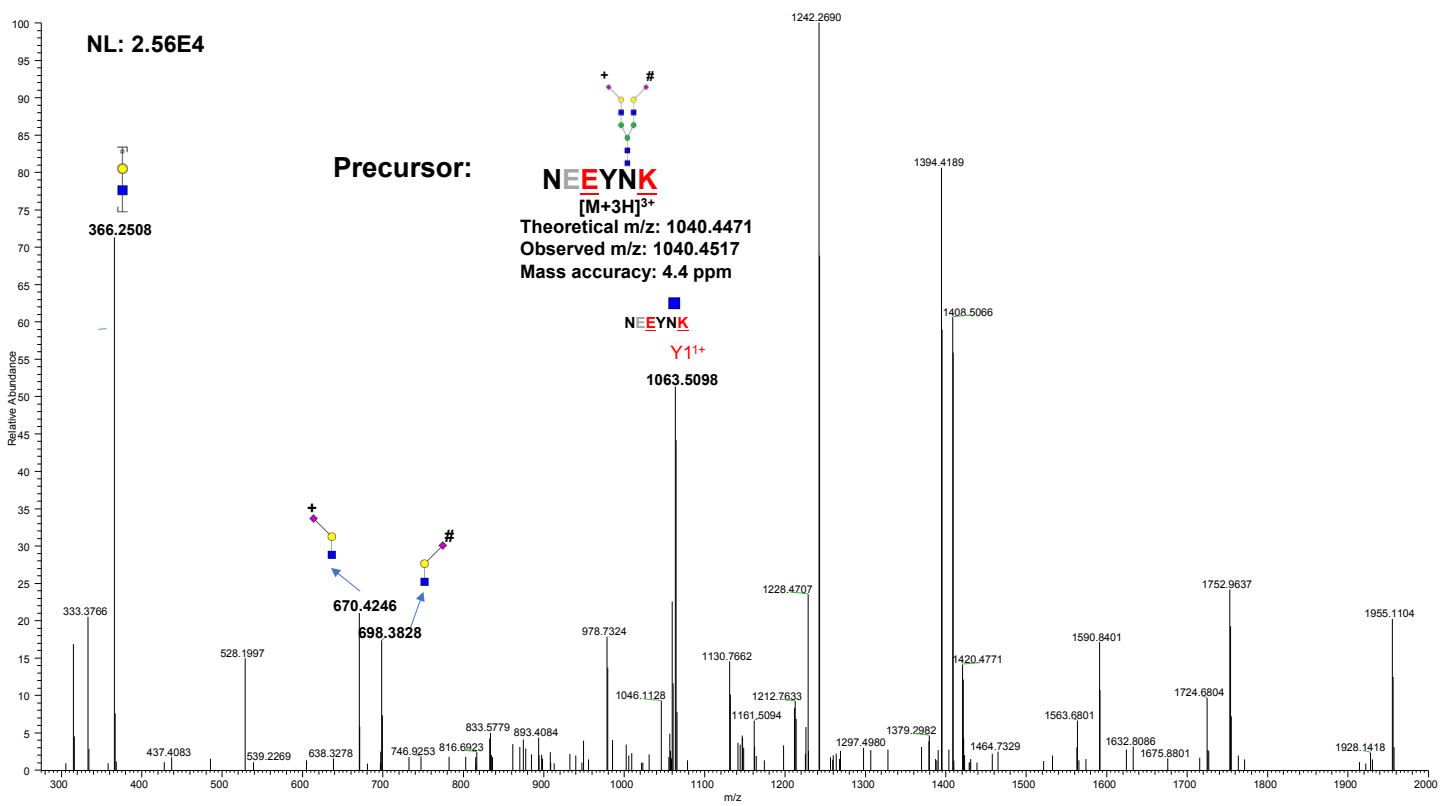

Figure S10. Representative MS/MS spectrum of DOSG-derivatized N-glycopeptides derived from AGP. Symbols as in Scheme 2. 


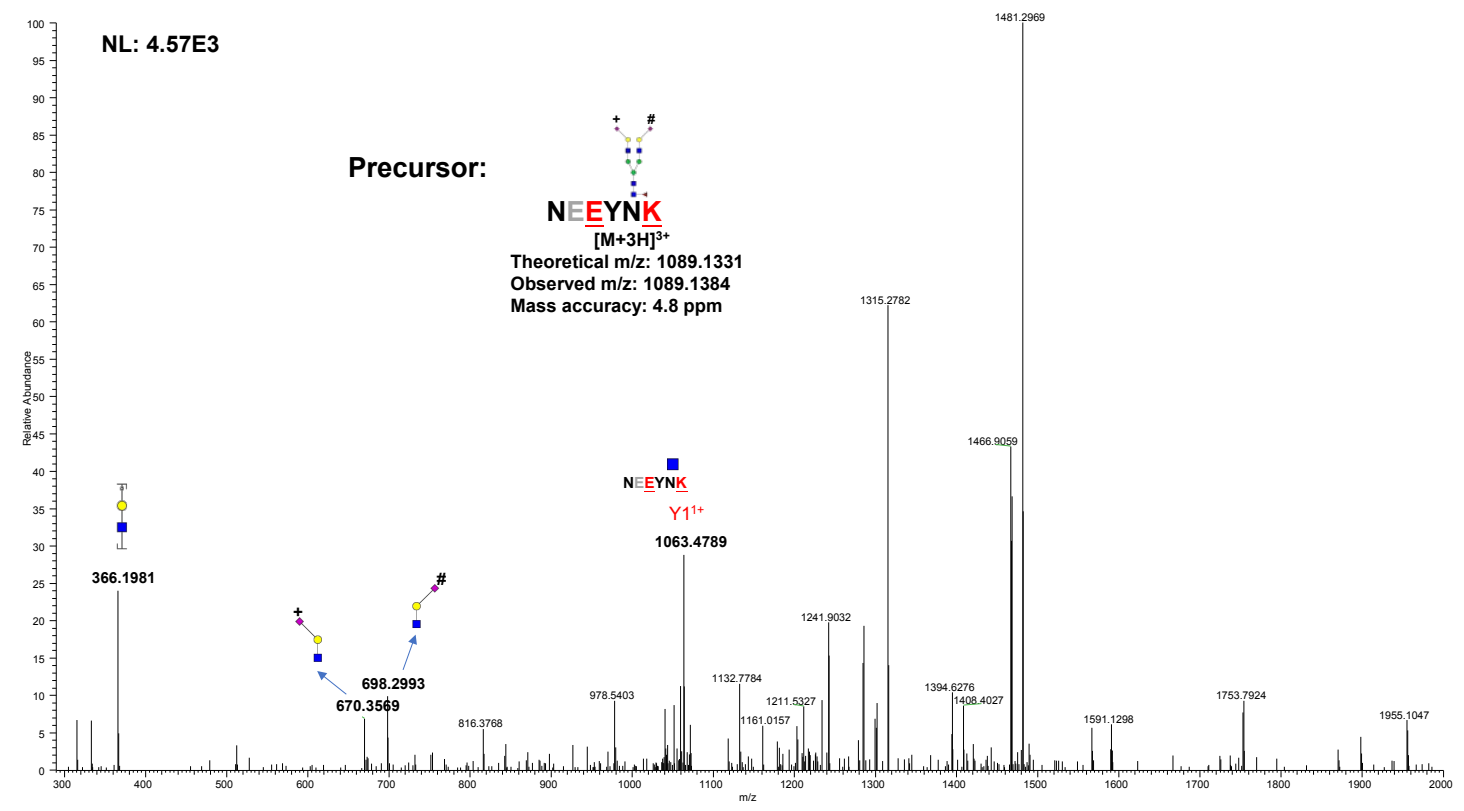

Figure S11. Representative MS/MS spectrum of DOSG-derivatized N-glycopeptides derived from AGP. Symbols as in Scheme 2. 


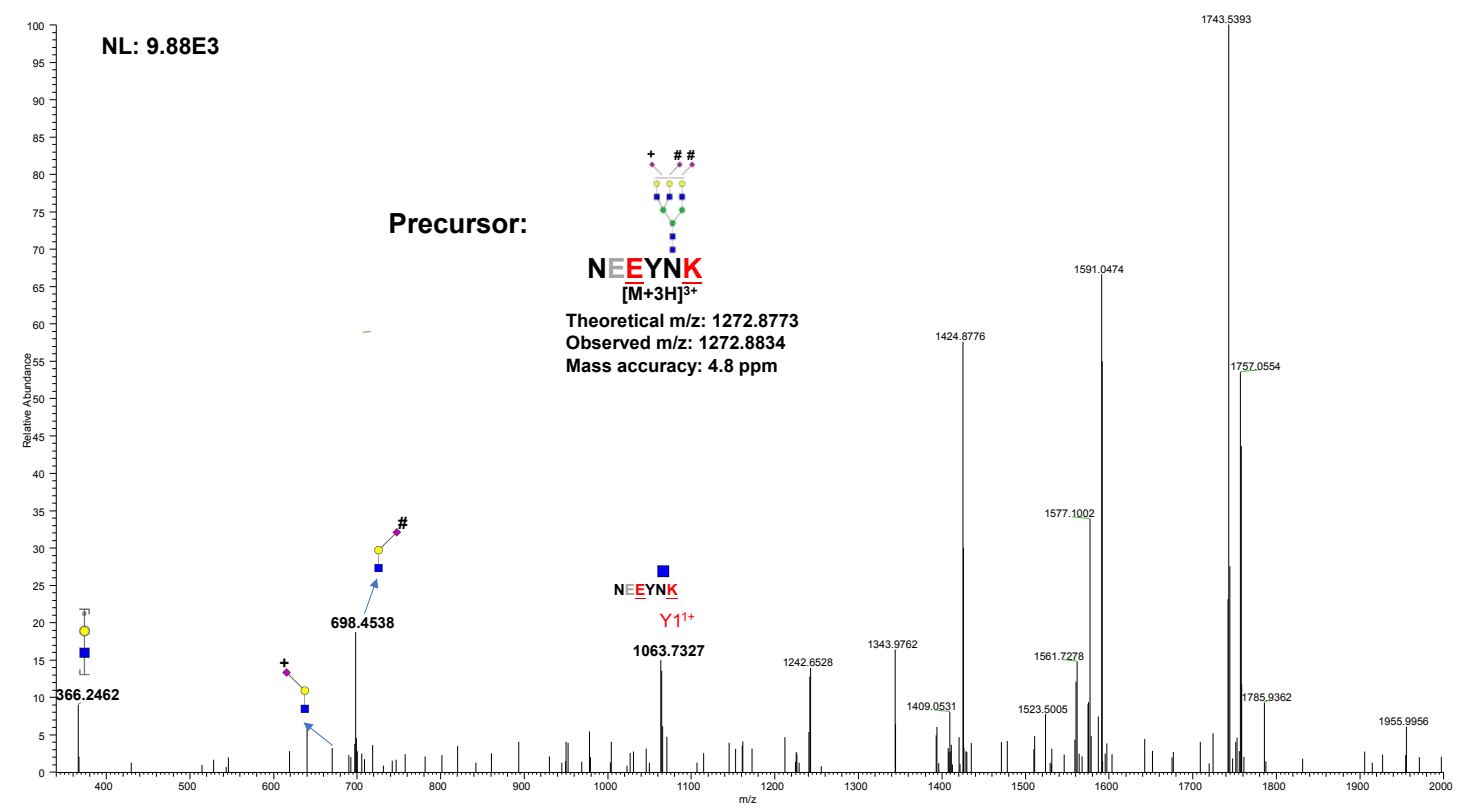

Figure S12. Representative MS/MS spectrum of DOSG-derivatized N-glycopeptides derived from AGP. Symbols as in Scheme 2. 


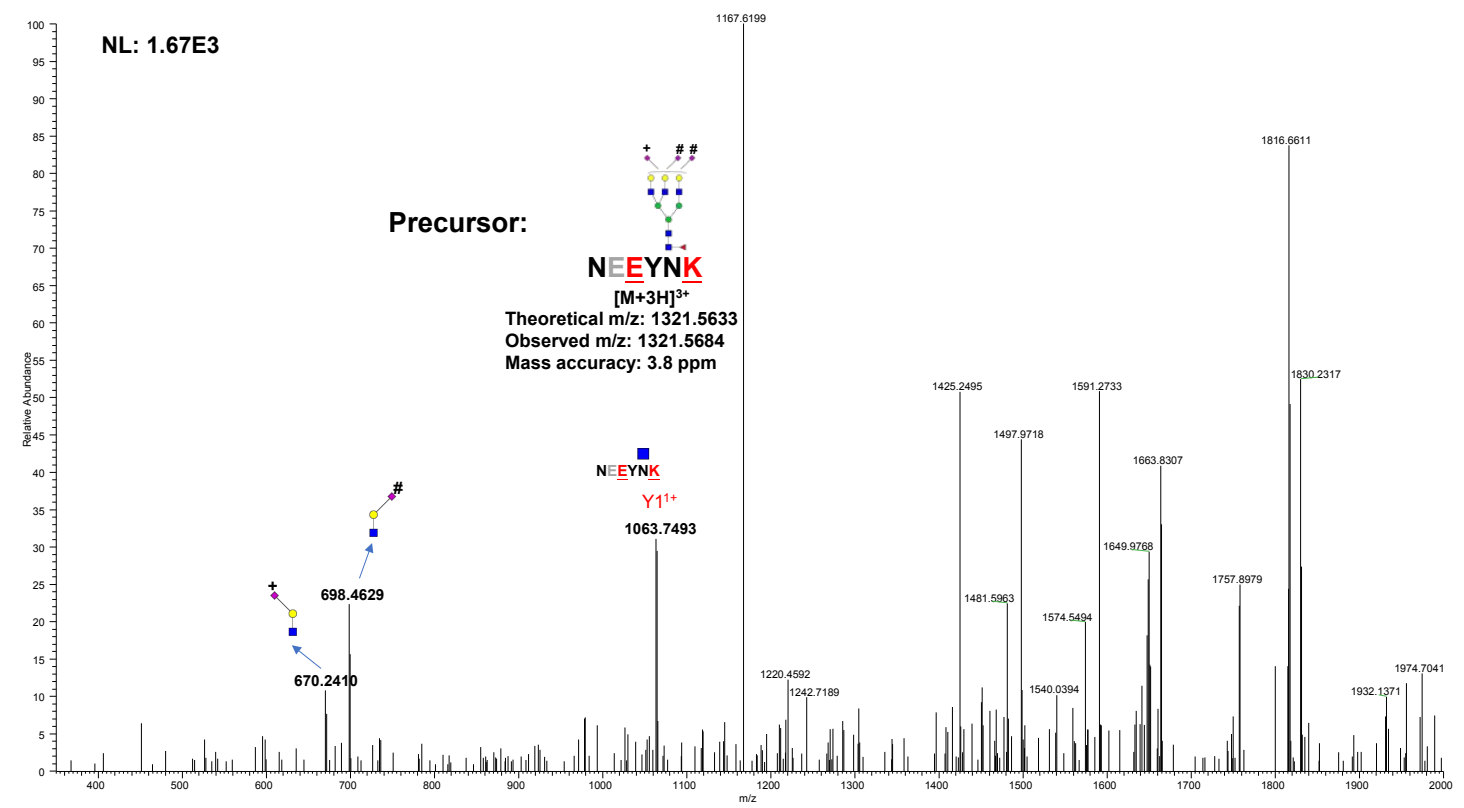

Figure S13. Representative MS/MS spectrum of DOSG-derivatized N-glycopeptides derived from AGP. Symbols as in Scheme 2. 


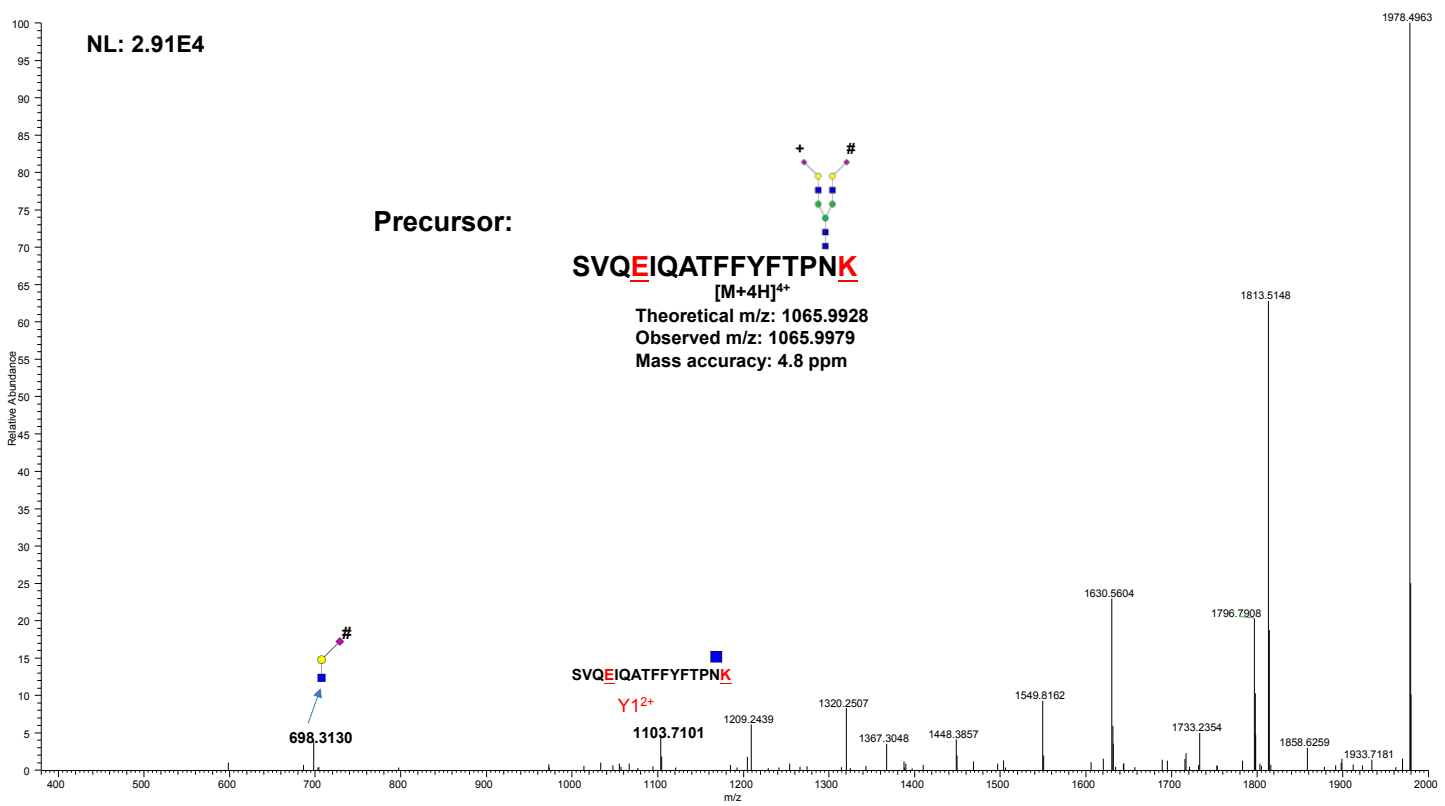

Figure S14. Representative MS/MS spectrum of DOSG-derivatized N-glycopeptides derived from AGP. Symbols as in Scheme 2. 


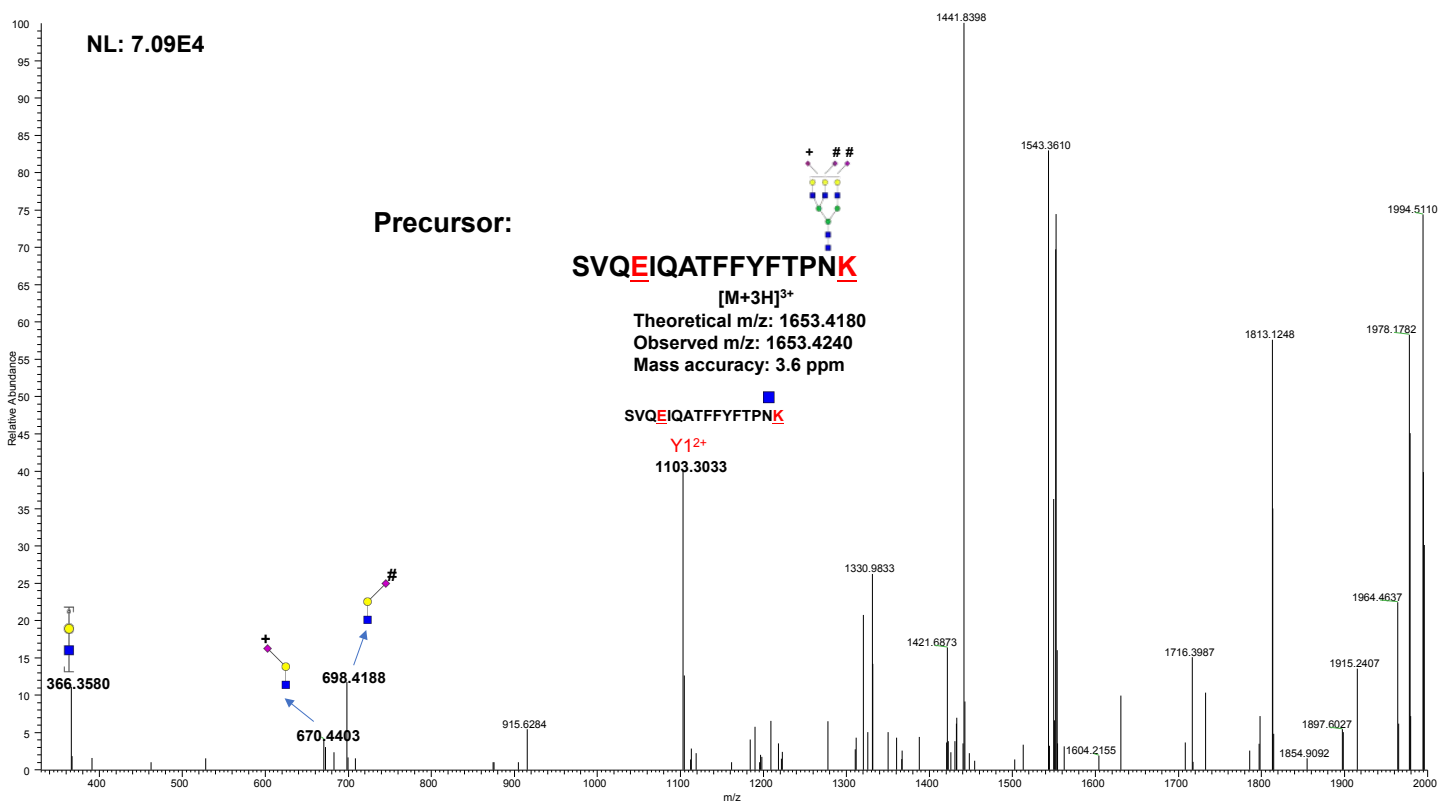

Figure S15. Representative MS/MS spectrum of DOSG-derivatized N-glycopeptides derived from AGP. Symbols as in Scheme 2. 


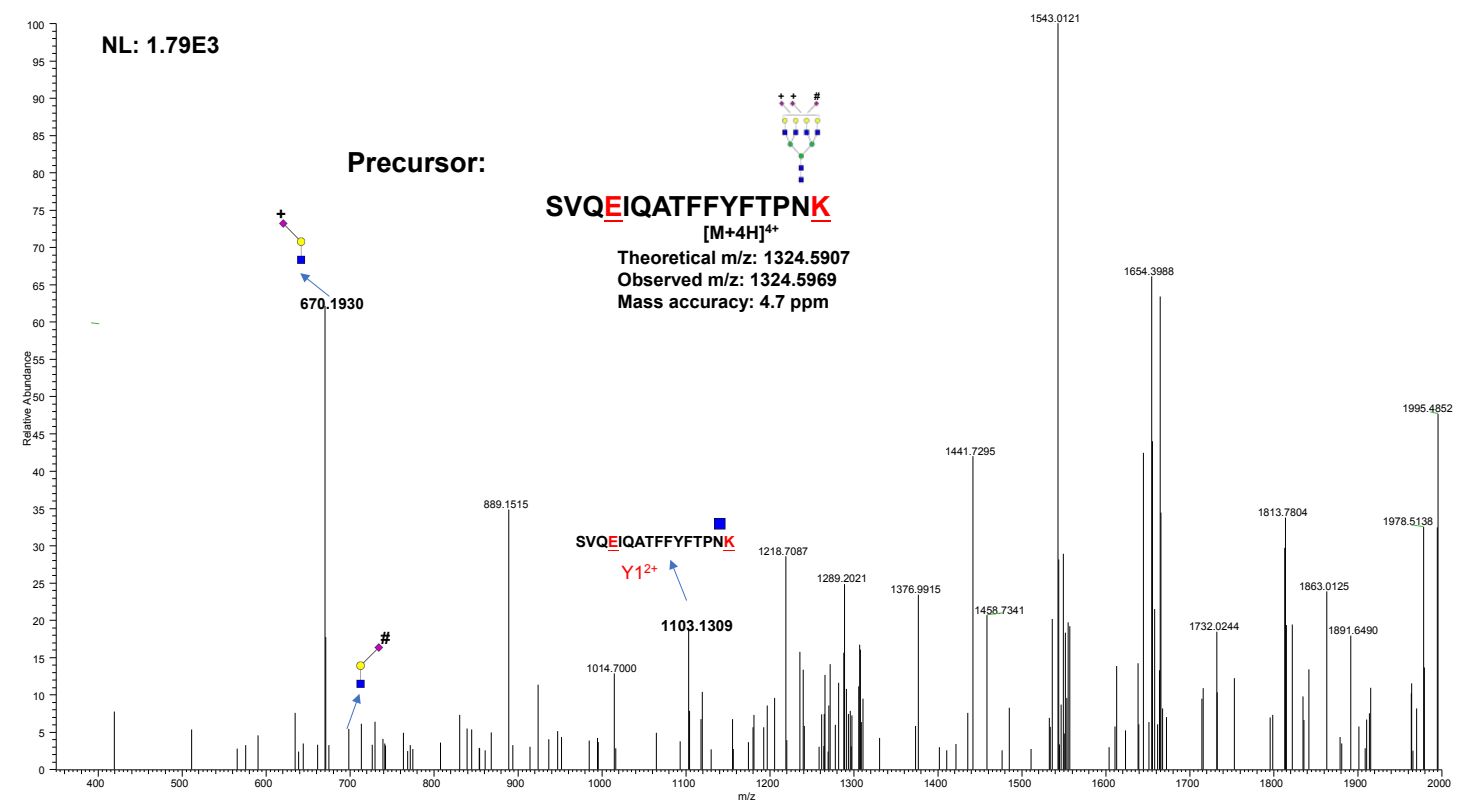

Figure S16. Representative MS/MS spectrum of DOSG-derivatized N-glycopeptides derived from AGP. Symbols as in Scheme 2. 


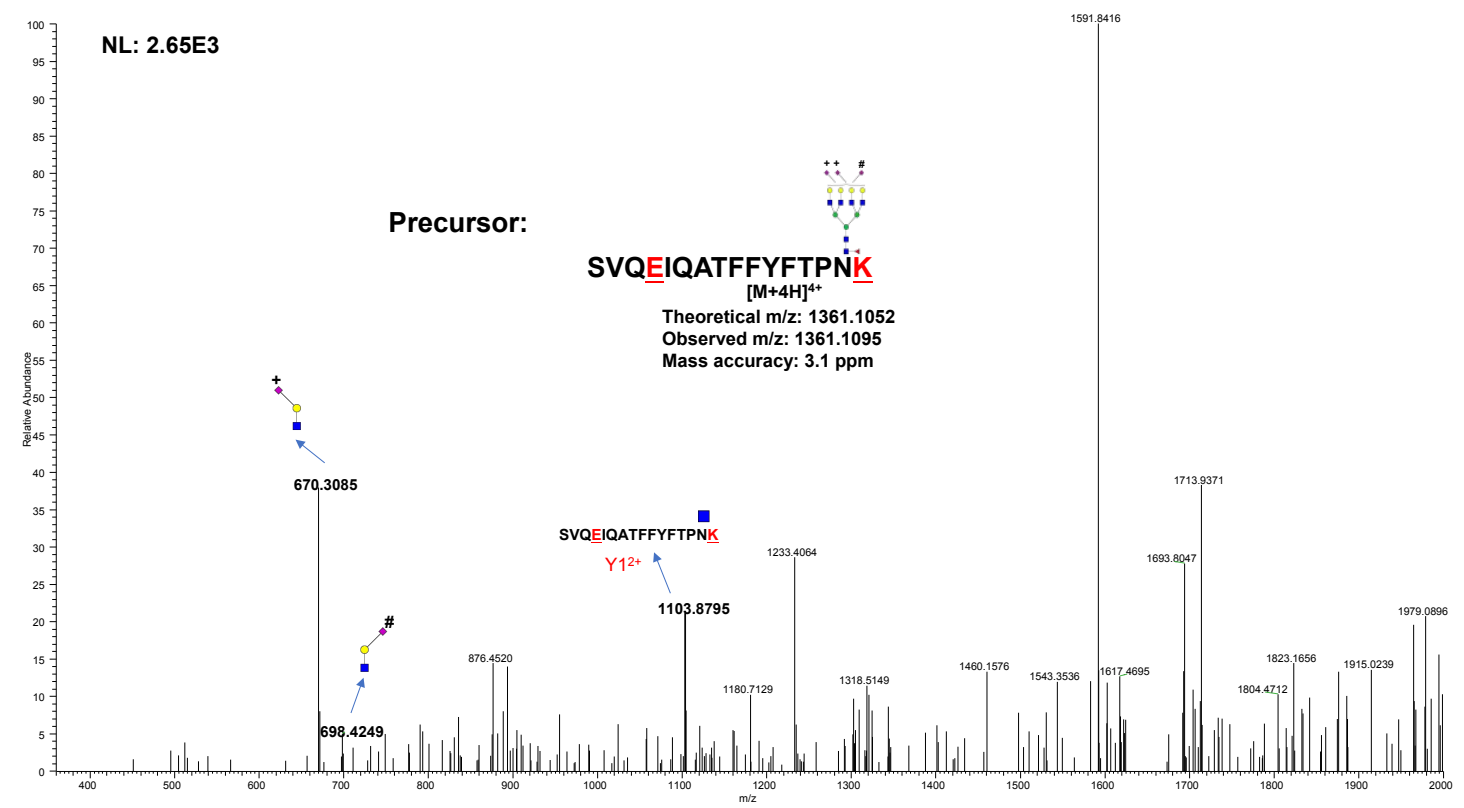

Figure S17. Representative MS/MS spectrum of DOSG-derivatized N-glycopeptides derived from AGP. Symbols as in Scheme 2. 


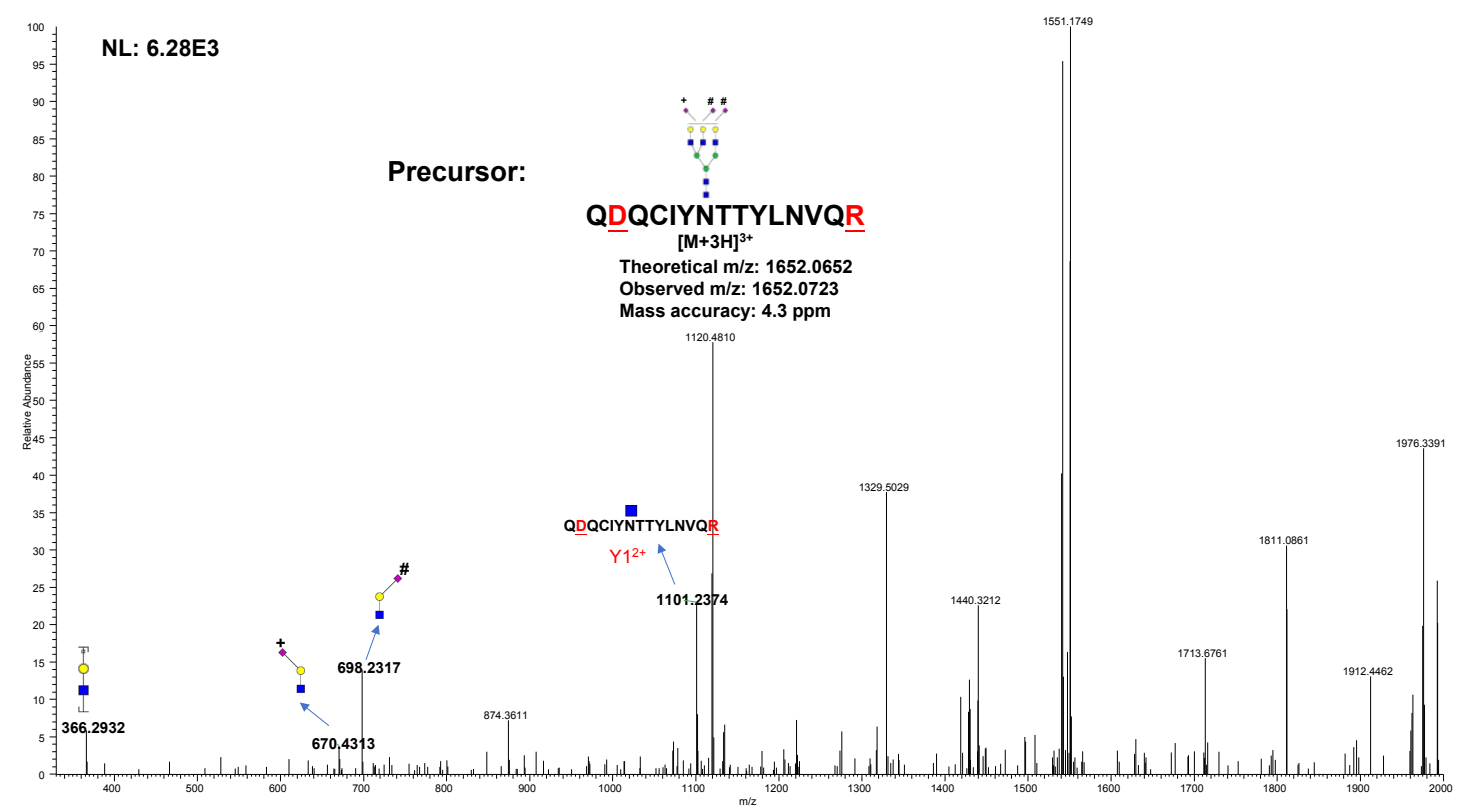

Figure S18. Representative MS/MS spectrum of DOSG-derivatized N-glycopeptides derived from AGP. Symbols as in Scheme 2. 


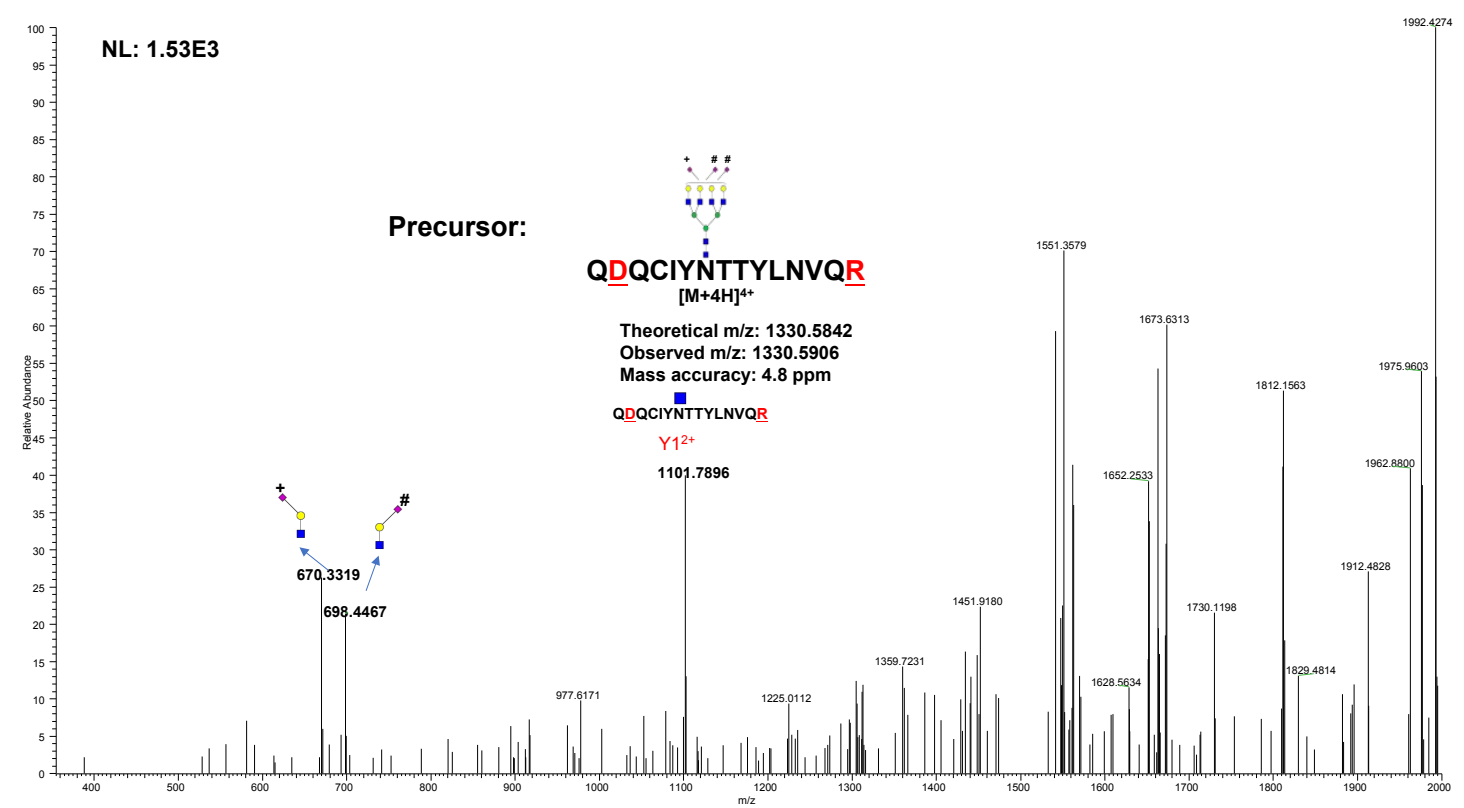

Figure S19. Representative MS/MS spectrum of DOSG-derivatized N-glycopeptides derived from AGP. Symbols as in Scheme 2. 


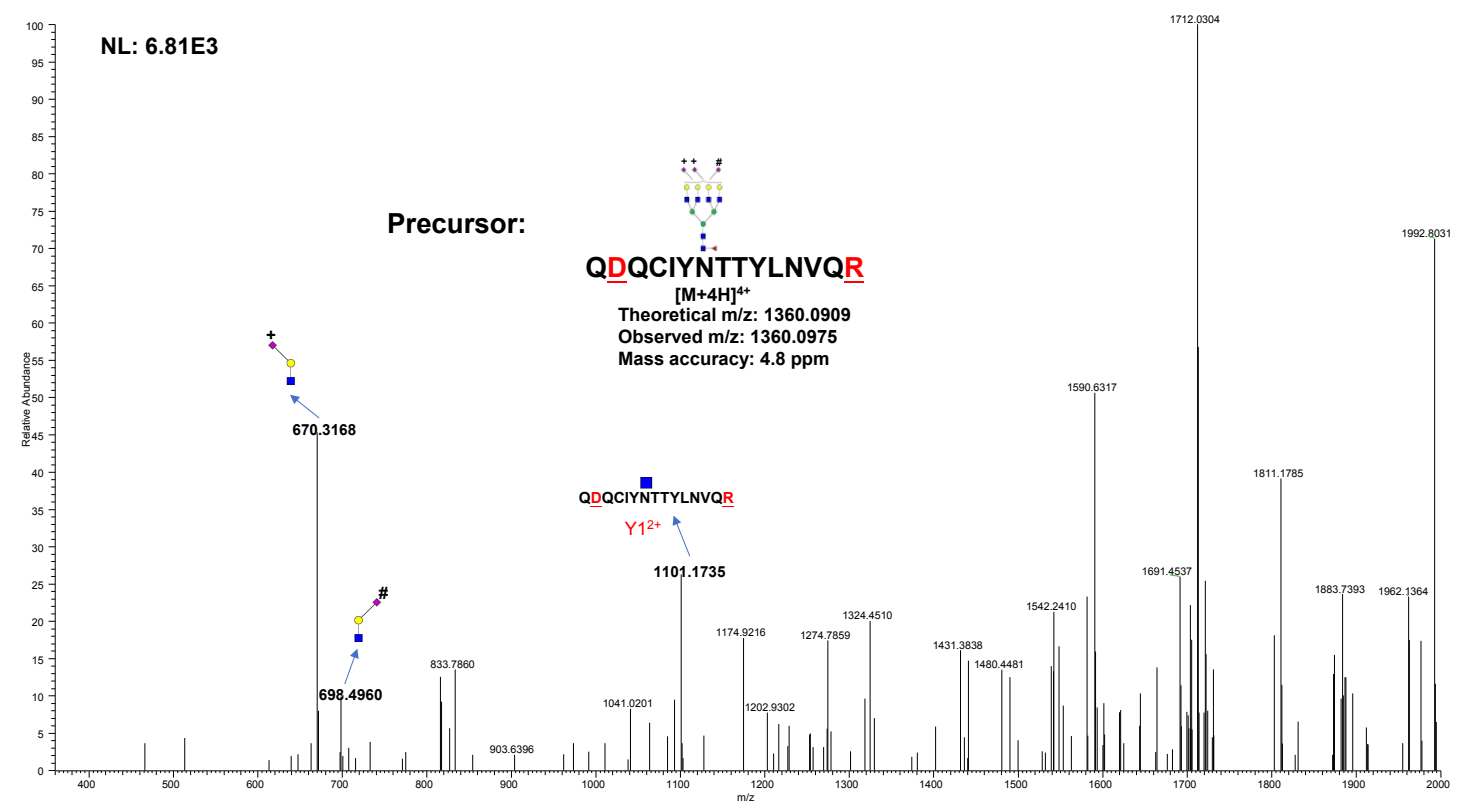

Figure S20. Representative MS/MS spectrum of DOSG-derivatized N-glycopeptides derived from AGP. Symbols as in Scheme 2. 


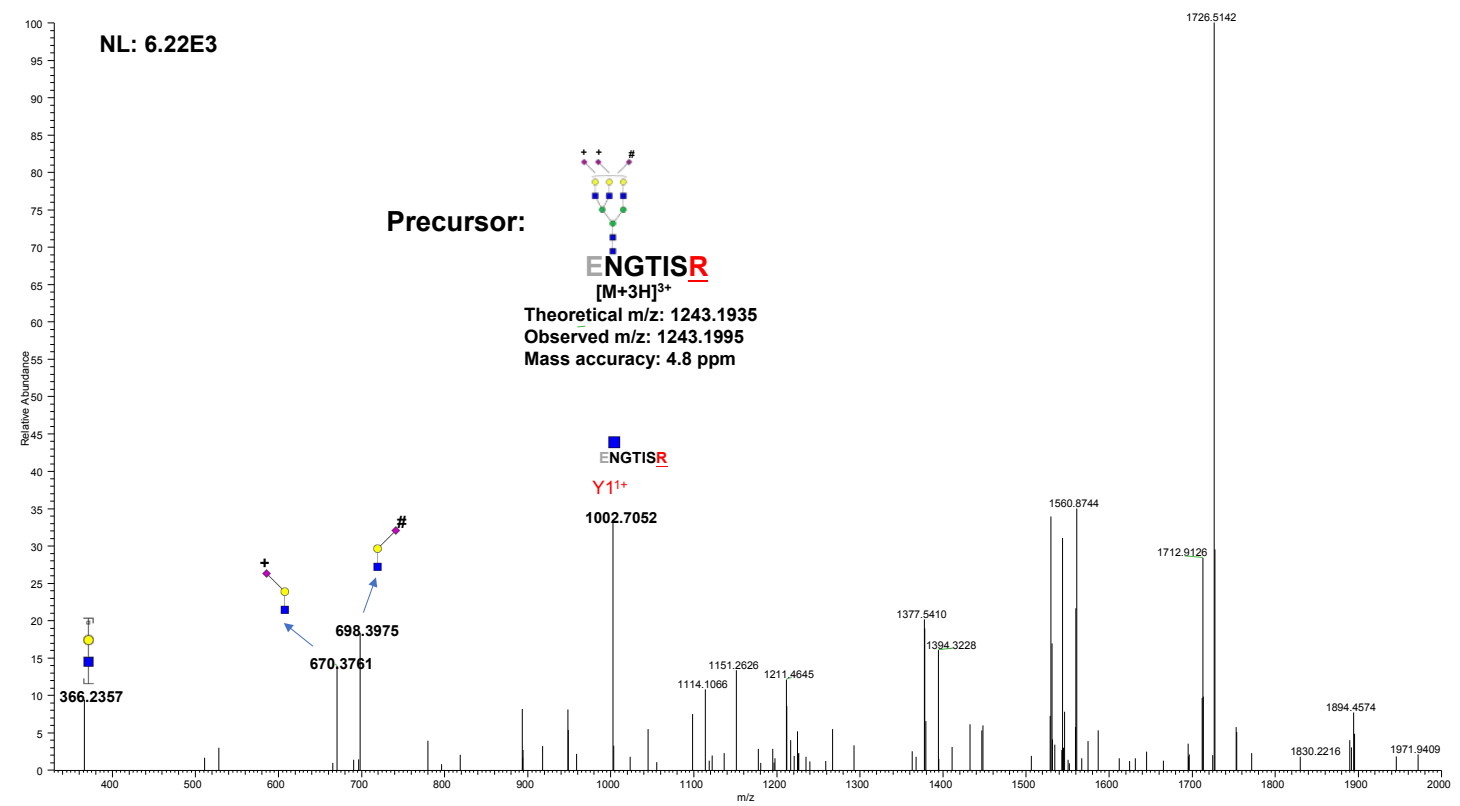

Figure S21. Representative MS/MS spectrum of DOSG-derivatized N-glycopeptides derived from AGP. Symbols as in Scheme 2. 


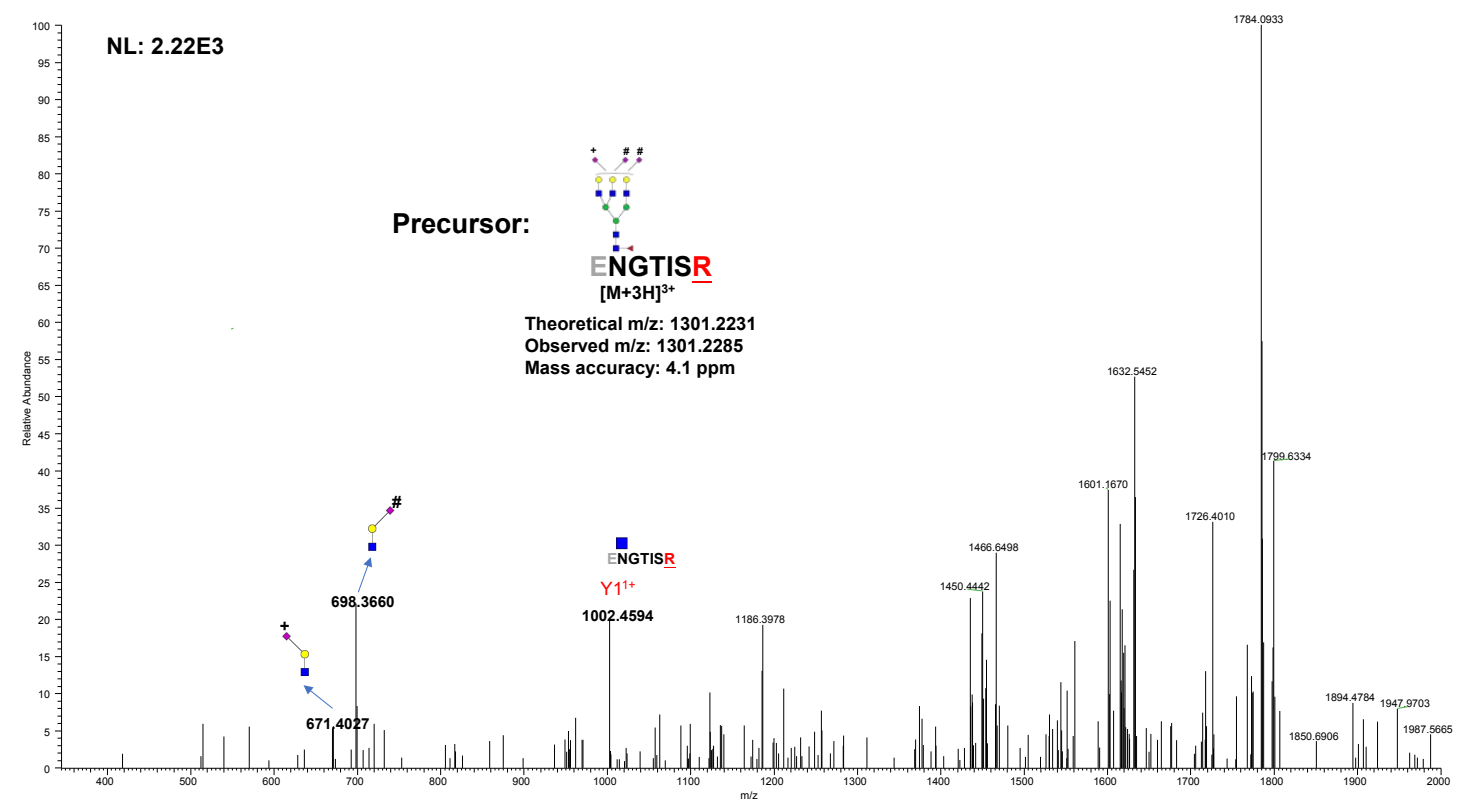

Figure S22. Representative MS/MS spectrum of DOSG-derivatized N-glycopeptides derived from AGP. Symbols as in Scheme 2. 


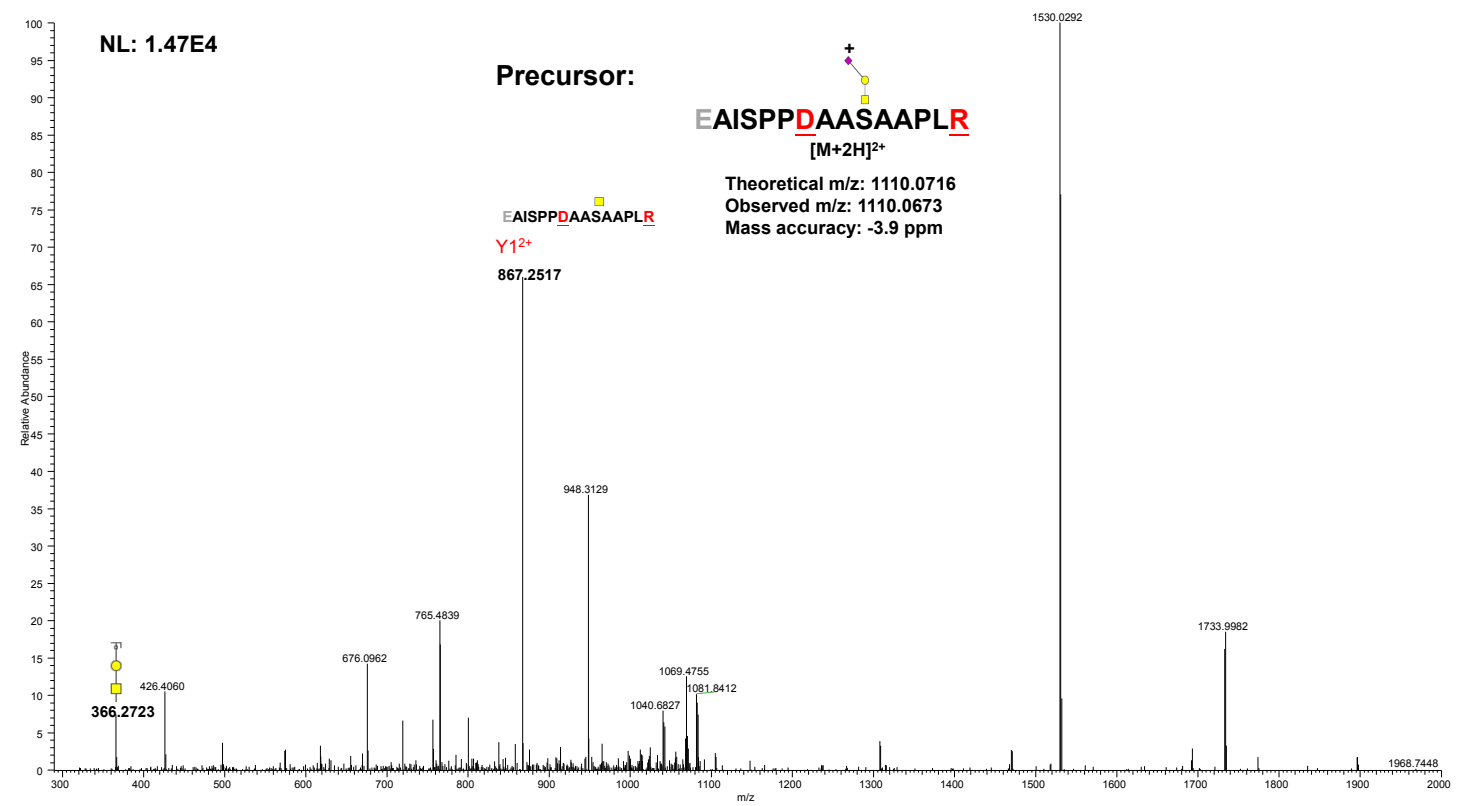

Figure S23. The MS/MS spectrum of DOSG-derivatized O-glycopeptides derived from EPO. Symbols as in Scheme 2. 


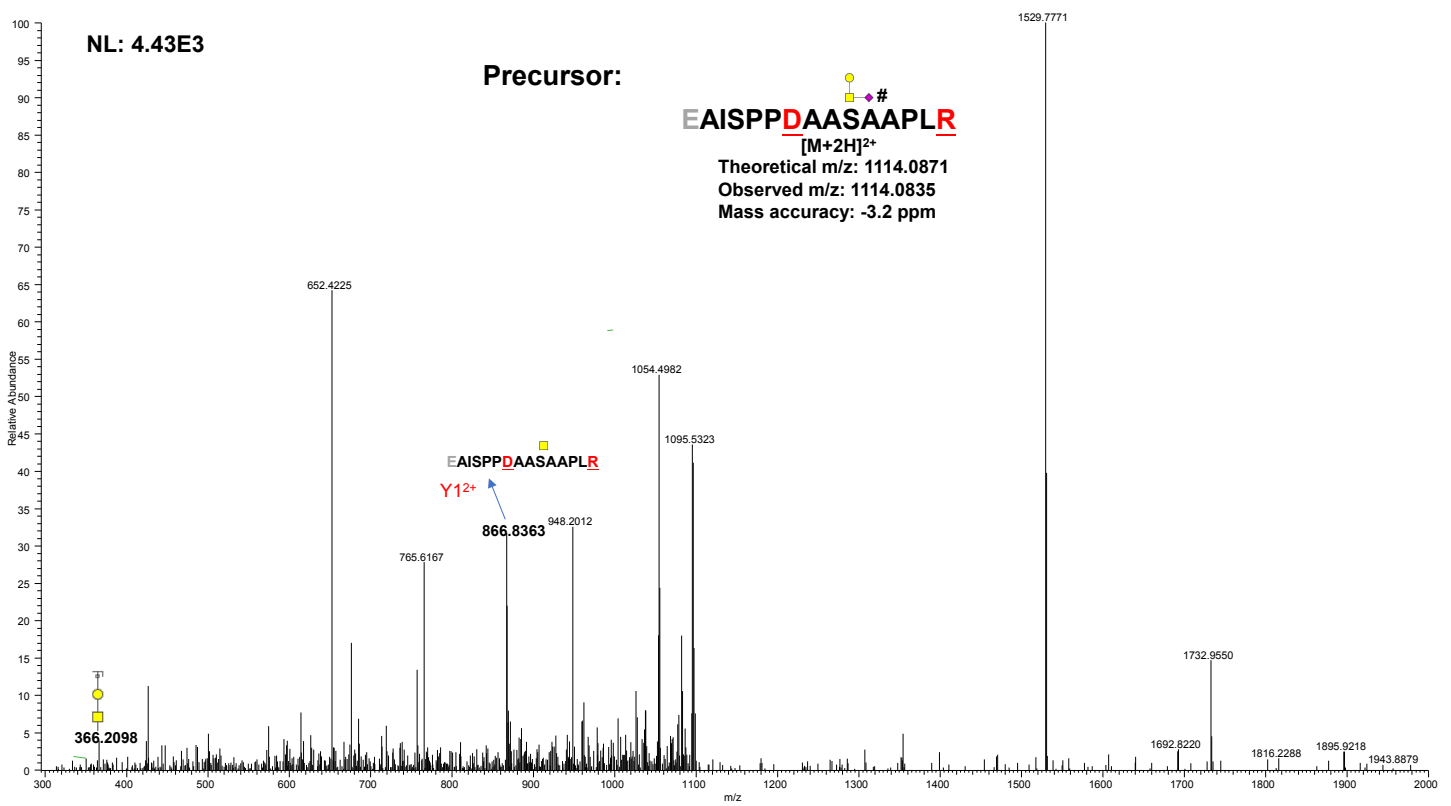

Figure S24. The MS/MS spectrum of DOSG-derivatized O-glycopeptides derived from EPO. Symbols as in Scheme 2. 


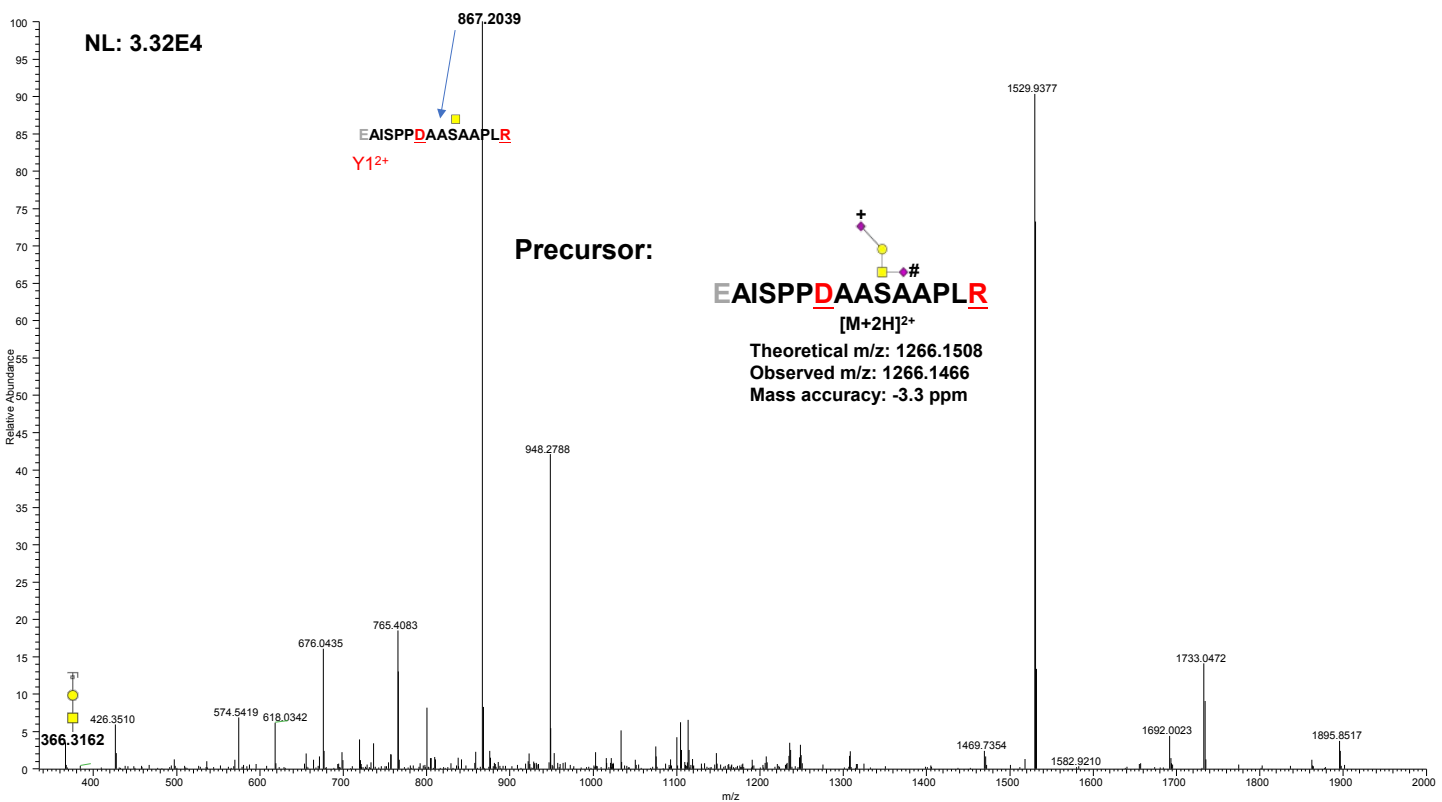

Figure S25. The MS/MS spectrum of DOSG-derivatized O-glycopeptides derived from EPO. Symbols as in Scheme 2. 


\section{References}

(1) Giansanti, P.; Tsiatsiani, L.; Low, T. Y.; Heck, A. J. Six alternative proteases for mass spectrometry-based proteomics beyond trypsin. Nat Protoc. 2016, 11, 993-1006.

(2) de Haan, N.; Reiding, K. R.; Haberger, M.; Reusch, D.; Falck, D.; Wuhrer, M. Linkage-specific sialic acid derivatization for MALDI-TOF-MS profiling of IgG glycopeptides. Anal Chem. 2015, 87, 8284-8291.

(3) Yang, W.; Shah, P.; Hu, Y.; Toghi Eshghi, S.; Sun, S.; Liu, Y.; Zhang, H. Comparison of Enrichment Methods for Intact N- and O-Linked Glycopeptides Using Strong Anion Exchange and Hydrophilic Interaction Liquid Chromatography. Anal Chem. 2017, 89, 11193-11197. 